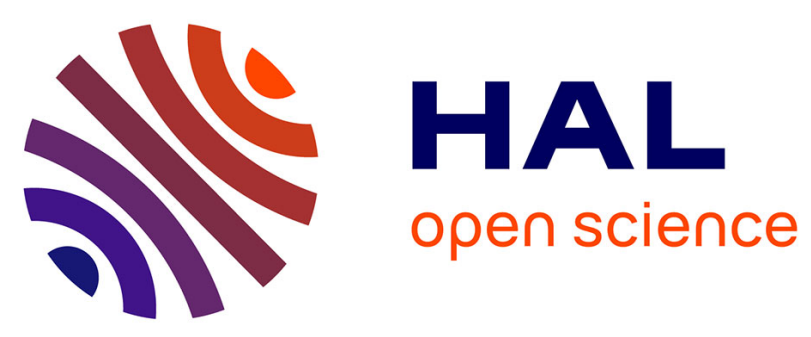

\title{
Kondo effect in real metals
}

Ph. Nozières, Annie Blandin

\section{- To cite this version:}

Ph. Nozières, Annie Blandin. Kondo effect in real metals. Journal de Physique, 1980, 41 (3), pp.193211. 10.1051/jphys:01980004103019300 . jpa-00209235

\section{HAL Id: jpa-00209235 https://hal.science/jpa-00209235}

Submitted on 1 Jan 1980

HAL is a multi-disciplinary open access archive for the deposit and dissemination of scientific research documents, whether they are published or not. The documents may come from teaching and research institutions in France or abroad, or from public or private research centers.
L'archive ouverte pluridisciplinaire HAL, est destinée au dépôt et à la diffusion de documents scientifiques de niveau recherche, publiés ou non, émanant des établissements d'enseignement et de recherche français ou étrangers, des laboratoires publics ou privés. 


\title{
Kondo effect in real metals
}

\author{
$\mathrm{Ph}$. Nozières \\ Institut Laue-Langevin, 156X, 38042 Grenoble Cedex, France
}

and A. Blandin

Laboratoire de Physique des Solides, Université Paris-Sud, 91405 Orsay, France

(Reçu le 21 septembre 1979, accepté le 30 octobre 1979)

\begin{abstract}
Résumé. - Dans le cadre d'un hamiltonien d'Anderson généralisé, nous étudions le comportement d'une impureté magnétique dans un métal réel, compte tenu de la structure orbitale des électrons localisés sur l'impureté, du champ cristallin et du couplage spin-orbite. Nous nous limitons à un schéma atomique, dans lequel l'impureté a une valeur bien définie entière (une transformation de Schrieffer Wolff est alors possible).

Les principales étapes d'une procédure de renormalisation sont décrites en détail: Au fur et à mesure que la température diminue, les états excités de la configuration atomique fondamentale se découplent l'un après l'autre. Nous analysons la hiérarchie de ces découplages, et leur interaction avec les singularités Kondo. Dans la mesure où un modèle de liquide de Fermi s'applique lorsque $T \rightarrow 0$, le nombre de paramètres indépendants est considérablement réduit par des arguments de symétrie et d'universalité qui court-circuitent la description numérique de la région de transition.

Cette première partie établit un langage. Nous l'appliquons au cas où l'état fondamental atomique est un singulet orbital. En l'absence d'anisotropies, les seuls paramètres sont le spin de l'impureté $S$ et le nombre $n$ de canaux orbitaux. Nous montrons l'existence d'un point fixe anormal (à couplage fini) lorsque $n>2 S$; ce point fixe est instable vis-à-vis d'une anisotropie. Nous discutons les trajectoires de renormalisation dans le cas d'un champ cristallin cubique pour différents choix de valence; nous précisons dans quelle mesure le comportement à basse température est universel.

Une analyse similaire est effectuée pour une impureté contenant un seul électron (ou trou) localisé. Nous discutons l'effet du champ cristallin et du couplage spin-orbite, et nous précisons leur influence sur le découplage Kondo et sur l'universalité.
\end{abstract}

\begin{abstract}
Starting from the most general form of the Anderson hamiltonian, the behaviour of magnetic impurities in real metals is considered, taking into account the orbital structure of the local impurity electrons, crystal field and spin orbit splittings. The analysis is carried out in an atomic limit, in which the impurity has a well defined integer valency (a Schrieffer Wolff transformation is then valid).

The main steps of a scaling procedure are described in detail. As the temperature goes down, the excited states of the ground state configuration decouple one after the other. The hierarchy of these decouplings, and their interplay with Kondo singularities are analyzed. When a Fermi liquid picture applies as $T \rightarrow 0$, the number of independent parameters may be reduced considerably using symmetry and universality arguments which bypass the numerical description of the crossover region.

That first part sets a language in which to describe specific problems. We apply that language to the case where the atomic ground state is an orbital singlet. In the absence of anisotropies, the only parameters are the impurity spin $S$ and the number of orbital channels $n$. We show that an anomalous fixed point occurs at finite coupling when $n>2 S$. That fixed point is unstable with respect to anisotropies. The scaling trajectories are discussed for a cubic crystal field for several choices of valencies. The universality of the low temperature behaviour is clarified.

A similar analysis is carried out when the atomic ground state only has one electron (or hole). The influence of crystal field and spin orbit interactions is analyzed - and their relevance to the Kondo crossover and to universality is ascertained.
\end{abstract}

1. Introduction. - Most papers dealing with the Kondo effect in metals are concerned with an idealized model as simple as possible, in order to focus attention on the interesting many body effects. Usually, no account is taken of the orbital structure of the impurity; the latter is assumed to have only a 
spin degree of freedom. One may either use the standard Kondo s-d model with a pure spin $S$ affixed to the impurity, or use the Anderson model for fictitious $l=0$ electrons $:$ in both cases, the actual nature of a transition impurity is ignored completely. That makes the theory simpler - but it renders any comparison with experiment somewhat hazardous.

In real life, the impurity localized states have a finite orbital angular momentum $l$. The corresponding generalization of the Anderson model was worked out long ago - yet in an incomplete form despite numerous successive corrections [1]. A completely general description, including spin orbit effects, crystal field splitting, etc., was given by Hirst [2], who analyzed in detail the group theoretical nature of the problem. Yet, until very recently, no attempt was made to study the Kondo effect in such realistic models. The first attempt was that of Yoshimori [3], who considered the Anderson model commonly found in the literature, involving only a Coulomb energy $U$ and an exchange energy $J$ on the impurity site. Using the perturbation approach of Yamada et al. [4], he derived a number of Ward identities satisfied by the various vertices, thus obtaining the low temperature properties of the system. Actually, his approach is not entirely correct, as it starts with an Anderson hamiltonian which is not the most general one consistent with the symmetry of the problem : one might fear that some of his results might follow from that restricted choice. A more general formulation of the problem, in the same language, has been given by Mihaly and Zawadowski [5] : it turns out that Yoshimori's results are indeed correct, independent of the unwarranted simplification he used.

In the present paper, we wish to show that the above results may be obtained very simply, without any fancy field theory or diagrams. The low temperature properties of a magnetic impurity are naturally described in the language of a Landau Fermi liquid theory, developed by one of us [6] in the simple case of a pure spin impurity. Here, we wish to extend such a description to realistic systems, such as a transition or rare earth impurity in a normal matrix. We shall see that a lot can be said without any numerical calculation, by using arguments that rely only either on symmetry, or on universality requirements (the result of Yoshimori is one of that type). The method is so simple that it can be used for more complicated situations, involving for instance crystal field splitting, spin orbit coupling, etc. In this way, one may hope to get closer to physical reality. The essential steps of such an economy class scaling are surveyed in ref. [7] for the standard pure spin Kondo impurity. Here we shall use the same approach for real metals. Our hope is to study the chemistry of the Kondo effect, to disentangle the hierarchy of all relevant physical effects, to see how the problem simplifies in various limiting cases. Since there are many parameters, an exhaustive discussion would by necessity be very long. Rather, we shall try to emphasize the salient ideas, to set a language that can be put to use in order to interpret a given experiment.

The basic Schrieffer Wolff transformation (which neglects valence fluctuations) is described in section 2 . The successive decoupling of atomic excited states (with unchanged valence) is treated in section 3 via a scaling approach. Universality in the $T=0$ limit is discussed in section 4 . Sections 5 and 6 provide specific examples, respectively the orbital singlet case, and atoms with a single localized electron (or hole).

2. General formulation of the problem. -2.1 THE ANDERSON MODEL. - Let us first ignore any anisotropy or spin orbit effect. The matrix is a normal metal, with plane wave eigenstates; the dispersion law $\varepsilon_{k}$ is isotropic. We add an impurity characterized by an atomic Hamiltonian $\boldsymbol{H}_{\mathrm{at}}$. We assume that these atomic states are constructed with one electron wave functions that have a well defined angular momentum $l$ ( $l=2$ in the transition series, $l=3$ for a rare earth). $H_{\text {at }}$ embodies arbitrarily complicated interactions between the atomic electrons. The impurity atom is coupled to the surrounding Fermi sea via the usual Anderson hybridization term. Let $C_{k \sigma}^{*}$ create a conduction electron with momentum $k$, spin $\sigma$, while $a_{m \sigma}^{*}$ creates an impurity electron with quantum numbers $m, \sigma$ (the number $l$, the same for every state, is understood). We write the Hamiltonian in the standard form

$\mathcal{H}=\varepsilon_{k} C_{k \sigma}^{*} C_{k \sigma}+H_{\mathrm{at}}+V_{k} C_{k \sigma}^{*} Y_{l m}(\hat{k}) a_{m \sigma}+$ c.c.

(1) is such as to preserve invariance under separate rotation of either the spins or the orbital space. Note that in writing (1), we already made a number of simplifications : besides ignoring crystal field and spin orbit coupling, we neglected electron interaction in the matrix, or between the matrix and the impurity. Such terms are usually thought to be small - but we should remember that they exist.

If we start from a real atomic problem, $H_{\mathrm{at}}$ involves only two particle interactions : its most general form then depends on a few parameters : the one electron atomic energy $\varepsilon_{\mathrm{d}}$ (measured from the Fermi energy which we choose as the origin), and $(l+1)$ Slater coefficients that describe the interaction energy. In the literature, it is customary to use a truncated model, which involves only two interaction parameters, a Coulomb energy $U$ and an exchange energy $J$. The corresponding interaction energy in $H_{\mathrm{at}}$ is written as $\left({ }^{1}\right)$

$$
\begin{aligned}
{\left[\frac{U}{2}-\frac{J}{4}\right] a_{m \sigma}^{*} a_{m^{\prime} \sigma^{\prime}}^{*} a_{m^{\prime} \sigma^{\prime}} a_{m \sigma} } & -J(\mathbf{S})_{\sigma \tau} \cdot(\mathbf{S})_{\sigma^{\prime} \tau^{\prime}} \times \\
& \times a_{m \sigma}^{*} a_{m^{\prime} \sigma^{\prime}}^{*} a_{m^{\prime} \tau^{\prime}} a_{m \tau}
\end{aligned}
$$

((2) is clearly invariant under spin and orbital rotation). In such a form, the interaction is diagonal in $m$.

( $\left.{ }^{1}\right)$ The $S_{\alpha}$ are the ordinary spin $1 / 2$ Pauli matrices. 
The theory of Yoshimori [3] is based on that particular choice. Actually, there is no reason why such a simplification should hold : the other Slater coefficients will introduce interaction terms of the form

$$
a_{m_{1} \sigma_{1}}^{*} a_{m_{2} \sigma_{2}}^{*} a_{m_{3} \sigma_{3}} a_{m_{4} \sigma_{4}}
$$

where only the global conservation conditions

$$
m_{1}+m_{2}=m_{3}+m_{4}, \quad \sigma_{1}+\sigma_{2}=\sigma_{3}+\sigma_{4}
$$

are obeyed. Taking these terms into account was precisely the goal of Mihaly and Zawadowski [4].

Altogether, the relevant parameters will be (i) the position of the resonance $\varepsilon_{\mathrm{d}}$, (ii) the $(l+1)$ Slater coefficients describing the interaction, (iii) the width of the resonance $\Delta=\pi \rho|V|^{2}$ (where $\rho$ is the density of states at the Fermi level for one spin direction).

The situation is not much changed if we add only spin orbit coupling : $\boldsymbol{H}_{\text {at }}$ becomes more complicated, but the other two terms in (1) are unmodified, because of rotational invariance together with the Kramers degeneracy. In contrast, taking the crystalline potential into account makes the problem markedly more complicated. First of all, the Fermi surface of the host material may become anisotropic - and even multivalued for a transition metal. Moreover, the hybridization matrix element

$$
V_{k, m} C_{k \sigma}^{*} a_{m \sigma}
$$

will now depend on the direction of $k$ in a complicated way. Hence an apparently hopeless mess ! Fortunately, the situation is not as bad as it looks, since the impurity can only sense the conduction electrons through the self energy matrix $\left({ }^{2}\right)$

$$
\Delta_{m m^{\prime}}(\varepsilon)=\pi \sum_{k} V_{k m}^{*} V_{k m^{\prime}} \delta\left(\varepsilon-\varepsilon_{k}\right) .
$$

For a given point group symmetry around the impurity, we can decompose the $(2 l+1)$ one electron states into irreducible representations $\alpha$ (for instance, $l=2$ electrons in a cubic crystal field split into a triplet $T$ and doublet $E$ ). Invariance under the point group symmetry implies that $\Delta_{m m^{\prime}}$ factorizes into separate unit matrices for each representation $\alpha$. Anisotropies of the Fermi surface and of $V_{k}$ are thus lumped into different resonant widths $\Delta_{\alpha}$ for each representation $\left(\Delta_{\mathrm{T}}\right.$ and $\Delta_{\mathrm{E}}$ in the above example).

Besides changes in $\Delta_{\alpha}$, the crystal field acts to split the degeneracies of $H_{\mathrm{at}}$. The resulting eigenstates again correspond to specific representations of the impurity point group. The interplay of such splittings with the anisotropy of $\Delta$ has been studied thoroughly by Hirst [2], using a detailed group theoretical approach. Here we do not need to go into its detail : qualitative statements will be enough for our purpose.

$\left({ }^{2}\right)$ That would not be true if we included interactions of the conduction electron. For instance, degeneracies of the conduction band in a transition host would have drastic consequences.
We now return to (1), and we discuss in some detail the hierarchy in the eigenstates of $H_{\text {at }}$. Going down the scale of successively smaller splittings, we proceed as follows :

- The larger energy differences correspond to different valencies, i.e. number $n_{\mathrm{d}}$ of electrons localized on the impurity. Since the conduction band acts as a reservoir, we deal here with free energies, $E-\mu N$ (put another way, one electron energies are measured from the Fermi level). Let $n_{\mathrm{d}_{0}}$ be the valency of the ground state, $\varepsilon_{0}$ the subspace of atomic states with that valency. The hybridization term in (1) couples $\varepsilon_{0}$ to the subspaces $\varepsilon_{ \pm}$with valencies $n_{\mathrm{d}_{0}} \pm 1$. Typical ionization free energies $E_{ \pm}$from $\varepsilon_{0}$ to $\varepsilon_{ \pm}$are usually $\sim 10 \mathrm{eV}$ (mixed valence compounds being just those for which $E_{ \pm}$is accidentally small).

- Within a given valency subspace, the various terms are split according to Hund's rule. The ground state of $H_{\mathrm{at}}$ is obtained by first maximizing the total spin $S$, and then its total orbital angular momentum $L$. Typical splittings may be of a few eV.

- For transition metal impurities, the next step in the ladder is crystal field splitting - typically a fraction of an $\mathrm{eV}$. An orbital multiplet $(L \neq 0)$ will split into irreducible representations of the impurity point group. The resulting lowest component may be itself an orbital singlet or multiplet. For instance, for d-electrons in a cubic field, the triplet one electron state T is usually lower than E. Following Hund's rule, the ground state of $H_{\text {at }}$ is an orbital singlet for $n_{\mathrm{d}}=3(S=3 / 2)$ and for $n_{\mathrm{d}}=8(S=1)$. If the ground state is a multiplet, it will be eventually split by the spin orbit coupling $(\lesssim 1 \mathrm{eV})$.

- For a rare earth impurity, spin orbit occurs first, the $(L S)$ multiplet splitting into states of different $J$. The crystal field splitting is rather smaller.

These various atomic energy scales, varying from $E_{ \pm} \sim 10 \mathrm{eV}$ down to a small fraction of an $\mathrm{eV}$, should be compared with the resonant width $\Delta$. The latter is fairly large in a transition metal $(\sim 0.1 \mathrm{eV})$, very small in a rare earth. $A$ priori, it is not obvious which of these ratios is relevant. We shall see in the next section that $\Delta$ is important only when compared to the largest scale $E_{ \pm}$, related to a change in valency.

\subsection{EliminATION OF IONIZED CONFIGURATIONS :} THE SCHRIEFFER WOLFF TRANSFORMATION. - If $\Delta \ll E_{ \pm}$, we can treat the hybridization term in (1) within second order perturbation theory. We thereby construct an effective Hamiltonian (more exactly a retarded self energy), acting on the subspace $\varepsilon_{0}$. Let us for simplicity assume that $V_{k}$ is constant (this is in no way essential). We further define

$$
\sum_{k} C_{k \sigma} Y_{l m}(\hat{k})=C_{m \sigma}
$$

(despite its index $m, C_{m \sigma}$ describes s-wave conduction 
electrons - but in an $(l, m)$ combination). Lumping $(m, \sigma)$ into a single index $\alpha$, we may write the self energy as

$$
H_{\mathrm{eff}}=H_{\mathrm{at}}^{0}+T_{\alpha \alpha^{\prime}}^{+} C_{\alpha}^{*} C_{\alpha^{\prime}}+T_{\alpha \alpha^{\prime}}^{-} C_{\alpha^{\prime}} C_{\alpha}^{*}
$$

$H_{\mathrm{at}}^{0}$ is the projection of $H_{\mathrm{at}}$ on the subspace $\varepsilon_{0}$. The quantities $T_{\alpha \alpha^{\prime}}^{ \pm}$are atomic operators, acting on that same subspace

$$
\left.\begin{array}{l}
T_{\alpha \alpha^{\prime}}^{+}=V^{2} a_{\alpha} \frac{1}{z-H_{\mathrm{at}}} a_{\alpha^{\prime}}^{*} \\
T_{\alpha \alpha^{\prime}}^{-}=V^{2} a_{\alpha^{\prime}}^{*} \frac{1}{z-H_{\mathrm{at}}} a_{\alpha}
\end{array}\right\}
$$

$z$ is the energy of interest, ultimately the ground state energy of $H_{\mathrm{at}}$.

Eq. (5) results from virtual ionization of the impurity, respectively to $\mathcal{E}^{ \pm}$; it describes inelastic scattering of conduction electrons (the latter changes its channel $\alpha$, while the impurity jumps to another state within $\varepsilon_{0}$ ).

If we disregard an irrelevant shift in atomic energies, we may write (5) in the equivalent form

$$
\begin{aligned}
H_{\text {eff }} & =H_{\mathrm{at}}^{0}+T_{\alpha \alpha^{\prime}} C_{\alpha}^{*} C_{\alpha^{\prime}} \\
T_{\alpha \alpha^{\prime}} & =T_{\alpha \alpha^{\prime}}^{+}-T_{\alpha \alpha^{\prime}}^{-} .
\end{aligned}
$$

The complexity is hidden in $T_{\alpha \alpha^{\prime}}$, whose shape depends on the various atomic splittings within $\varepsilon_{0}$, $\varepsilon_{+}$and $\varepsilon_{-}$. A simple limit obtains if these intravalency splittings are small when compared to $E_{ \pm}$. Then the energy denominators in (5) are constant, and $T_{\alpha \alpha^{\prime}}$ reduces to

$$
\begin{aligned}
T_{\alpha \alpha^{\prime}} & =-\frac{V^{2}}{E_{+}} a_{\alpha} a_{\alpha^{\prime}}^{*}+\frac{V^{2}}{E_{-}} a_{\alpha^{\prime}}^{*} a_{\alpha} \\
& =\left\{-\frac{V^{2}}{E_{+}} \delta_{\alpha \alpha^{\prime}}+V^{2}\left[\frac{1}{E_{+}}+\frac{1}{E_{-}}\right]\right\} a_{\alpha^{\prime}}^{*} a_{\alpha}
\end{aligned}
$$

(remember that $T$ acts only on the subspace $\varepsilon_{0}$ ). Except for the first term which corresponds to pure potential scattering, the operator (7) depends only on a single parameter : it has a built in symmetry, which we shall denote as "VS » symmetry (Valency Splitting only). That symmetry holds exactly if both $\varepsilon_{0}$ and $\varepsilon_{ \pm}$are degenerate (for instance, a ground state $n_{\mathrm{d}_{\mathrm{o}}}=1$ virtually excited to $n_{\mathrm{d}}=0$, in the absence of crystal field or spin orbit effects); in the more general case, it is only approximate when $E_{+}$and $E_{-}$are large. Small departures from VS symmetry are easily characterized by expanding $T_{\alpha \alpha^{\prime}}$ in powers of

$$
\delta H_{\mathrm{at}}=H_{\mathrm{at}}-E_{ \pm} .
$$

They will reflect splittings of the intermediate states.

Eq. (7) is only a first step in the usual Schrieffer Wolff transformation : as of now, we retain all the impurity states belonging to $\varepsilon_{0}$, not just the lowest multiplet. We shall see in the next section how to pro- ceed to the next step, i.e. eliminating the excited states of $\varepsilon_{0}$ by a further perturbation expansion. Right now, let us look more closely at the validity of (7). It implies, of course, that the ionized configurations are not excited thermally : $T \ll E_{ \pm}$. Moreover, the virtual admixture of these configurations into a given state of $\varepsilon_{0}$ must carry a negligible norm, a requirement that implies $\left({ }^{3}\right)$ :

$$
\begin{aligned}
\sum_{k} \sum_{\alpha} V^{2}\left[\left(1-n_{k}\right)\left\langle a_{\alpha}^{*}\left(\frac{1}{z-H_{\mathrm{at}}-\varepsilon_{k}}\right)^{2} a_{\alpha}\right\rangle+\right. \\
\left.+n_{k}\left\langle a_{\alpha}\left(\frac{1}{z-H_{\mathrm{at}}+\varepsilon_{k}}\right)^{2} a_{\alpha}^{*}\right\rangle\right] \ll 1 .
\end{aligned}
$$

Usually, the conduction band width is $\gtrsim E_{ \pm}$. Making the replacement $\sum_{k} \rightarrow \rho \int \mathrm{d} \varepsilon_{k}$, we see that the condition (8) is qualitatively equivalent to

$$
\frac{\Delta}{\pi}\left[\frac{n_{\mathrm{d}_{0}}}{E_{-}}+\frac{M-n_{\mathrm{d}_{0}}}{E_{+}}\right] \ll 1
$$

(where $M=2(2 l+1)$ is the total number of channels). Thus, the existence of many orbital channels enhances the valence fluctuations [9], which may become large even if $\Delta$ is appreciably smaller than $E_{ \pm}$. That fact must be kept in mind when assessing the validity of a Schrieffer Wolff transformation.

3. Decoupling of atomic excited states. 3.1 LOGARITHMIC SINGULARITIES : A SCALING APPROACH. - A perturbation treatment of the scattering Hamiltonian (6) leads to logarithmic singularities at zero temperature, as shown by Kondo in the ordinary s-d model. The simplest way to cope with such singularities is to use a scaling approach, along the lines laid out by Anderson [10] and Wilson [11]. One progressively eliminates part of the conduction band states by introducing a symmetric cut off $\pm D$ around the Fermi level. As $D$ is reduced, the effective hamiltonian is renormalized in such a way as to leave the physics unchanged. The various one electron couplings are the relevant variables that depend logarithmically on $D$. While growing, they trigger the appearance of higher order terms (multi-electron interactions and retardation effects), which make the actual dynamics quite complicated. The mechanism of such a scaling procedure was surveyed recently by one of us [7] for the ordinary s-d model $(l=0)$. The main conclusions remain valid here. In a first transient region, details of the band shape are important; on the other hand once $D$ is small enough compared to the original band width, the scaling trajectory depends only on a

$\left({ }^{3}\right)$ Our definition of the spherical harmonics is such that the angular average

$$
\left\langle Y_{l m}(\hat{k}) Y_{l m^{\prime}}(\hat{k})\right\rangle=\delta_{m m^{\prime}}
$$

hence the condition $\alpha^{\prime}=\alpha$ in (8). 
few relevant parameters : anisotropies in $T_{\alpha \alpha^{\prime}}$ and splittings in $H_{\mathrm{at}}^{0}$. (The density of states $\rho$ may be taken as constant in the range $-D,+D$.)

In the pure spin case $(l=0)$, the scattering Hamiltonian (6) depends only on two parameters : potential and exchange scattering

$$
T_{\alpha \alpha^{\prime}}=T_{\sigma \sigma^{\prime}}=V \delta_{\sigma \sigma^{\prime}}+\Gamma \mathrm{S} \cdot \mathbf{s}_{\sigma \sigma^{\prime}}
$$

( $S$ is the impurity spin). In lowest order, $V$ does not change upon scaling, while $\Gamma$ obeys a universal differential equation

$$
\frac{\mathrm{d} \Gamma}{\mathrm{d} D}=\frac{1}{D}\left[-\rho \Gamma^{2}+\rho^{2} \Gamma^{3}+\cdots\right]=\frac{f(\rho \Gamma)}{\rho D} .
$$

The second order approximation to (10) is enough to find the Kondo temperature $T_{\mathrm{K}}$ at which $J$ crosses over from weak to strong coupling,

$$
T_{\mathrm{K}} \sim D_{0} \mathrm{e}^{-1 / \rho \Gamma_{0}}
$$

(where $D_{0}, \Gamma_{0}$ are the initial conditions). One feature is important : there is only one energy scale in the problem, $T_{\mathbf{K}}$.

In the presence of orbital effects, other energy scales appear, namely the level splittings of $\boldsymbol{H}_{\mathrm{at}}^{\mathbf{0}}$. They can cover a very broad range, from Hund's rule down to very small spin orbit splittings. If a given splitting $\Delta E_{0}$ is $\ll D$, we may ignore it. If instead it is $\gg D$, the corresponding excited level decouples from the scaling procedure : it may be discarded. The two limiting regimes are simple - albeit kinematically different. The cross over region $D \sim \Delta E_{0}$ is complicated (non logarithmic).

The central issue is now to compare $\Delta E_{0}$ with $T_{\mathrm{K}}$. Does the coupling grow before the excited state decouples $\left(T_{\mathrm{K}} \gg \Delta E_{0}\right)$, or after $\left(T_{\mathrm{K}} \ll \Delta E_{0}\right)$ ? The low temperature behaviour will be markedly different in the two cases. In the intermediate situation, $T_{\mathrm{K}} \sim \Delta E_{0}$, the two cross overs are mixed, and we do not expect any simple universal result. We should reconsider the question for every splitting $\Delta E_{0}$. In view of the variety of possible atomic level schemes, the zoology of behaviours is extremely broad, and we can only hope to give a few general guidelines.

Most of the important physical features are already apparent in the lowest second order approximation, the poor man's scaling of Anderson. The change upon scaling of the scattering amplitude $T_{\alpha \alpha^{\prime}}$, given by (6),

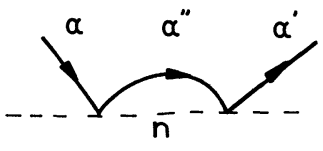

(a)

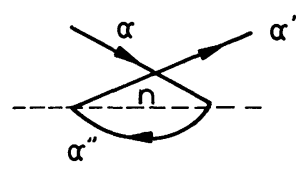

(b)
Fig. 1. - The two diagrams controlling the second order scaling of $T_{\alpha \alpha^{\prime}}$. The dotted line denotes the impurity state $n$ (inside the manifold $\varepsilon_{0}$ ). The full line is the free electron propagator (diagonal in the index $\alpha=(m, \sigma))$. follows from the two diagrams of figure 1. Inside these diagrams, the impurity (dotted line) can be in any state $|n\rangle$ belonging to $\varepsilon_{0}$, with energy $E_{n_{0}}$. The full line is the propagator for $C_{\alpha}$, which because of rotational invariance is diagonal in $\alpha$ :

$$
G_{\alpha \alpha^{\prime}}(z)=\delta_{\alpha \alpha^{\prime}} \sum_{k} G_{k}(z) .
$$

These scattering amplitudes are operators in the atomic subspace $\varepsilon_{0}$. If we reduce the cut off from $D$ to $D-\delta D$, we suppress intermediate states with $\varepsilon_{k}=+D$ in figure $1 a$, with $\varepsilon_{k}=-D$ in figure $1 b$. We must therefore correct the scattering matrix by the missing amount, which yields the following scaling equation

$$
\begin{aligned}
\frac{\partial T_{\alpha \alpha^{\prime}}}{\partial D}= & \sum_{\alpha^{\prime \prime}, n} T_{\alpha \alpha^{\prime \prime}} \frac{|n\rangle\langle n|}{D+E_{n_{0}}-z} T_{\alpha^{\prime \prime} \alpha^{\prime}}- \\
& -\sum_{\alpha^{\prime \prime}, n} T_{\alpha^{\prime \prime} \alpha^{\prime}} \frac{|n\rangle\langle n|}{D+E_{n_{0}}-z} T_{\alpha \alpha^{\prime \prime}} .
\end{aligned}
$$

In (11), $z$ is the total energy of the scattering stateelectron and impurity (at $T=0$, it will reduce to the ground state energy $E_{00}$ of $\varepsilon_{0}$ ).

Let us first assume that $D$ is much larger than all the excitation energies within $\varepsilon_{0}$. (Such an approximation is not very realistic for Hund's rule splittings : we take it as a limiting case.) For an electron with kinetic energy $\varepsilon_{k} \ll D$, it follows that $E_{n_{0}}-z \ll D$ : the splittings of $H_{\mathrm{at}}^{0}$ are thus completely irrelevant, and the scaling equation (11) takes the very simply form

$$
\frac{\partial T_{\alpha \alpha^{\prime}}}{\partial \log D}=\sum_{\alpha^{\prime \prime}}\left[T_{\alpha \alpha^{\prime \prime}}, T_{\alpha^{\prime \prime} \alpha^{\prime}}\right]
$$

We recover a logarithmic problem, with no apparent energy scale. All the states in $\varepsilon_{0}$ are treated on an equal footing, as if they were degenerate.

When $D$ (and the temperature) are much smaller than all the excitation energies of $H_{\mathrm{at}}^{0}$, an opposite situation holds. The summation in (11) is dominated by the ground state multiplet. We can ignore the contribution of excited states, which are totally decoupled. The effective scaling equation becomes

$$
\frac{\partial T_{\alpha \alpha^{\prime}}}{\partial \log D}=T_{\alpha \alpha^{\prime \prime}} P_{0} T_{\alpha^{\prime \prime} \alpha^{\prime}}-T_{\alpha^{\prime \prime} \alpha^{\prime}} P_{0} T_{\alpha \alpha^{\prime \prime}}
$$

where $P_{0}$ is the projector on the ground state multiplet. The problem is again logarithmic, but with a different kernel. The cross over between two regimes is clearly apparent. In the intermediate region, $D \sim \Delta E_{0}$, one must solve the full equation (11) : the dependence on $D$ is not logarithmic.

3.2 STRUCTURE OF THE SCATTERING OPERATOR. Let us consider in more detail the large $D$ region, governed by (12). To the extent that the VS symmetry 
holds, the scattering matrix depends only on two parameters

$$
T_{\alpha \alpha^{\prime}}=a \delta_{\alpha \alpha^{\prime}}+b a_{\alpha^{\prime}}^{*} a_{\alpha} .
$$

If we carry that expression into (12), we see that the $V S$ symmetry is preserved upon scaling. The two coefficients $a$ and $b$ obey the equations

$$
\left.\begin{array}{l}
\frac{\partial b}{\partial \log D}=-M \rho b^{2} \\
\frac{\partial a}{\partial \log D}=n_{\mathrm{d}_{0}} \rho b^{2}
\end{array}\right\} .
$$

(We use the fact that $\sum_{\alpha^{\prime \prime}} a_{\alpha^{\prime \prime}}^{*} a_{\alpha^{\prime \prime}}=n_{\mathrm{d}_{0}}$ when acting on the subspace $\varepsilon_{0}$ ). Consequently, the exchange scattering $b$ grows logarithmically, as in the ordinary Kondo effect. The potential scattering $a$ is given by

$$
a=-b \frac{n_{\mathrm{d}_{0}}}{M}+\text { const. . }
$$

From (14) and (7), we infer the Kondo temperature $T_{\mathrm{K}}$ at which $\rho b \sim 1$ :

$$
\left.\begin{array}{rl}
T_{\mathrm{K}} & \sim D_{0} \exp \left[-\frac{1}{M \rho b_{0}}\right] \\
M \rho b_{0} & =\frac{M \Delta}{\pi}\left[\frac{1}{E_{+}}+\frac{1}{E_{-}}\right]
\end{array}\right\} .
$$

If we compare (16) with (9), we see that $T_{\mathrm{K}}$ must be very small $\left(\ll D_{0}\right)$ if the Schrieffer Wolff transformations is to be valid. In such a case, we are likely to hit the splittings of $H_{\mathrm{at}}^{0}$ before we reach $T_{\mathrm{K}}$ : renormalization in the range $D \gtrsim \Delta E_{0}$ is small.

In addition to the VS symmetric part (7), the scattering matrix may contain corrections $\delta T_{\alpha \alpha^{\prime}}$ arising from atomic splittings in the subspaces $\varepsilon_{0}, \varepsilon_{ \pm}$. These corrections also change upon scaling; if they are small, we may linearize the corresponding growth equation (12) :

$$
\begin{aligned}
& \frac{\partial T_{\alpha \alpha^{\prime}}}{\partial \log D}=\rho b \times \\
& \quad \times \sum_{\alpha^{\prime \prime}}\left\{\left[\delta T_{\alpha \alpha^{\prime \prime}}, a_{\alpha^{\prime}}^{*} a_{\alpha^{\prime \prime}}\right]+\left[a_{\alpha^{\prime \prime}}^{*} a_{\alpha}, \delta T_{\alpha^{\prime \prime} \alpha^{\prime}}\right]\right\} .
\end{aligned}
$$

Unfortunately, the shape of $T_{\alpha \alpha^{\prime}}$ is not preserved.

Quite generally, $\delta T_{\alpha \alpha^{\prime}}$ depends on a finite number $P$ of coefficients $C_{\lambda}$. The scaling equations (17) turn into a linear system for $C_{\lambda}$

$$
\frac{\partial C_{\lambda}}{\partial \log D}=-\rho b S_{\lambda \lambda^{\prime}} C_{\lambda^{\prime}} .
$$

On combining with (14), we see that

$$
\frac{\partial C_{\lambda}}{\partial b}=\frac{S_{\lambda \lambda^{\prime}} C_{\lambda^{\prime}}}{M b} .
$$

We only need to find the eigenvalues of $S_{\lambda \lambda^{\prime}}$. If we choose a basis where $S$ is diagonal, then

$$
C_{\lambda} \sim\left(\frac{b}{b_{0}}\right)^{\frac{S_{\lambda}}{M}} C_{\lambda_{0}}
$$

Depending on the sign of $S_{\lambda}$, the correction terms may grow or decay. If $S_{\lambda}>M$, the corresponding correction may become quite large as the cross over proceeds. However, there may be constraints that require $S_{\lambda}>M$ : we could not demonstrate that guess.

We now proceed to the region $D \ll \Delta E_{0}$. If the above $T_{\mathrm{K}}$ is $\ll \Delta E_{0}$, no cross over has developed yet by the time the excited states of $\varepsilon_{0}$ decouple : The low $D$ scaling will start from the first order projected scattering operator

$$
\widetilde{T}_{\alpha \alpha^{\prime}}=P_{0} T_{\alpha \alpha^{\prime}}^{0} P_{0} .
$$

The VS symmetry is no longer stable upon scaling. Even if we start from the simple form (7), more complicated terms will appear in $\widetilde{T}_{\alpha \alpha^{\prime}}$ as scaling proceeds. The number of independent parameters $C_{\lambda}$ that characterize $T_{\alpha \alpha^{\prime}}$ is smaller than before (it is the number of states one can build with the ground state multiplet and one conduction electron). Yet, it is usually larger than 2, and new couplings do appear.

3.3 Relevant PaRameters AND Kondo TEMPERATURES. - Altogether, we see that there exist two mechanisms that can generate new terms of $T_{\alpha \alpha^{\prime}}$ beyond the simple VS expression.

(i) Splittings in ionized states, $\varepsilon_{ \pm}$. The corresponding corrections are of order $\Delta E_{ \pm} / E_{ \pm}$compared to the main term.

(ii) Progressive decoupling of the excited multiplets in the ground state manifold $\varepsilon_{0}$ of $H_{\text {at }}$. Such a logarithmic growth begins when $D \sim \Delta E_{0}$. The resulting corrections at cut off $D$ are of order

$$
\rho T \log \left(\Delta E_{0} / D\right) .
$$

At first, they are negligible compared to (i) - at very low temperatures $\left(\sim T_{\mathrm{K}}\right)$, they will always take over.

We note one important point : the ratio $\Delta / \Delta E_{0}$ never enters. Put another way, comparing the width of the resonance to atomic splittings within $\varepsilon_{0}$ is of no relevance, even if these splittings are very small (e.g., spin orbit).

Sometimes it may happen that such extra couplings $\delta T_{\alpha \alpha^{\prime}}$ do not exist. Consider for instance the simple case where the ground state of $H_{\mathrm{at}}^{0}$ is an orbital singlet, $L=0$, with total spin $S$. In the absence of crystal field effects, the hamiltonian must be invariant under both orbital and spin rotation. The only terms that obey these requirements are

$$
\tilde{T}_{\alpha \alpha^{\prime}}=\delta_{m m^{\prime}}\left[V+\Gamma \mathbf{S} . \mathbf{s}_{\sigma \sigma^{\prime}}\right]
$$


(see next section). Despite the existence of several orbital channels, $\widetilde{T}$ has the same form as in the $l=0$ case. $V$ and $\Gamma$ obey the same scaling equation (10), and the Kondo temperature is now

$\tilde{T}_{\mathrm{K}}=D_{0} \exp \left[-\frac{1}{\rho \Gamma_{0}}\right]=D_{0} \exp \left[-\frac{1}{2 b_{0}}\right]$.

Compared to $(16)$, a factor $(2 l+1)$ has disappeared in the exponent, essentially because $m$ is conserved in the interaction (18). As a result, $\widetilde{T}_{\mathrm{K}}$ may be very much smaller than $T_{\mathrm{K}}$. A Kondo cross over may exist even if the Schrieffer Wolff transformation is not valid (i.e. $\left.M \Delta \sim E_{ \pm}\right)$. Put another way, valence fluctuations are not incompatible with the Kondo effect. However, we pay a price : there is no well defined starting point analogous to an s-d model. We must describe the Kondo effect in the full formalism of the Anderson model, and we cannot separate that description in two distinct steps.

3.4 SuCCESSIVE CROSS OVERS. - As the cut off $D$ goes down, it sweeps through the various splittings of $H_{\text {at }}$. At each cross over, the kinematics of the impurity changes, as well as the nature of the scaling. Since the logarithmic evolution is very slow, these successive cross overs will be well separated only if the splittings have a very different order of magnitude. (Otherwise, one cannot disentangle the decoupling of different excited states.)

The first decoupling corresponds to Hund's rule. Only the lowest atomic multiplet, with angular momenta $L, S$, survives. The effective Hamiltonian is built on that $L S$ manifold. In practice, Hund's rule splittings are not very small compared to $E_{ \pm}$, and one can hardly identify that cross over. (Note that spin and orbital variables are linked by the Pauli principle : they decouple together.) Later on, crystal field and spin orbit splittings come into play. Since they may be much smaller, one may observe two distinct scaling regions (before and after the corresponding splitting). Ultimately, the Kondo effect will act only on the lowest state of $\varepsilon_{0}$.

Consider a given scaling range between two successive splittings, $\Delta E_{0 \alpha} \ll \Delta E_{0, \alpha-1}$. For that particular range, we may estimate a Kondo temperature $T_{\mathrm{K} \alpha}$. Two possibilities may occur

(i) $T_{\mathrm{K} \alpha} \ll \Delta E_{0 \alpha}$ : The couplings did not grow much when $D$ reaches the lower limit $\Delta E_{0 \alpha}$. The physics does not change appreciably in that range, and any Kondo cross over will occur later on.

(ii) $\Delta E_{0 \alpha}<T_{\mathrm{K} \alpha}<\Delta E_{0, \alpha-1}$. Then one crosses from weak to strong coupling in the range under consideration. The physics is completely changed by the time one attacks the next splitting $\Delta E_{0 \alpha}$ : a strong coupling analysis is required.

If the strong coupling ground state on the central site is non degenerate (as it is for the usual $L=0$, $S=1 / 2$ model), it is the end of the Kondo effect.
Nothing will happen as the cut off sweeps by smaller splittings $\Delta E_{0}$. On the other hand, if the new ground state is still degenerate, a new Kondo effect may emerge. The possibility of such a two step Kondo effect $\left({ }^{4}\right)$ was emphasized by Cornut and Coqblin [12] in the case of spin orbit splitting in rare earths. Many different behaviours are clearly possible, and a systematic discussion is impossible.

\subsection{NUMBER OF INDEPENDENT PARAMETERS AFTER} FULL SCHRIEFFER WOLFF DECOUPLING. - We first ignore crystal field and spin orbit effects, and we consider an atomic ground state of $H_{\mathrm{at}}^{0}$ with momenta $L, S$. The only operators in $T_{\alpha \alpha^{\prime}}$ that are invariant under both orbital and spin rotation are of the form

$$
(\mathbf{S . s})_{\sigma \sigma^{\prime}}^{p}(\mathbf{L} . \mathbf{l})_{m m^{\prime}}^{q}
$$

Because $s$ is a Pauli spin $1 / 2$ matrix, only the values $p=0,1$ are independent, irrespective of $S$ (it is the smallest of $S$ and $s$ that fixes the number of parameters). We recover the usual potential and spin exchange terms. Similarly, the number of $q$ values is

$$
|2 \operatorname{Min}(l, L)+1| \text {. }
$$

Altogether the Schrieffer Wolff hamiltonian will have

$$
\left\{\begin{array}{l}
2 \text { parameters if } L=0 \\
2(2 l+1) \text { parameters if } L \neq 0 .
\end{array}\right.
$$

(These parameters characterize the interaction energy in each of the states one can build with the impurity $L S$ and one electron.)

Such a conclusion contradicts the usual picture of atomic physics, in which the interaction energy inside an $l$-shell depends only on $(l+1)$ Slater parameters. It should be realized, however, that the origin of the interaction is quite different. In an Hartree Fock atom, one deals directly with the expectation value of a two particles interaction term; the Slater parameters arise in the angular decomposition of $e^{2} / r_{12}$.

The Schrieffer Wolff interaction, instead, originates in virtual ionization of the impurity, its strength being $\sim 1 / E_{ \pm}$. In order to characterize the various splittings, one needs the $(l+1)$ Slater parameters, plus the position $\left(\varepsilon_{l}-\mu\right)$ of the atomic level with respect to the Fermi energy. Strictly speaking, the dimension of the Schrieffer Wolff parameter space is therefore $(l+2)$, not $(4 l+2)$. However, if the excited state splittings, $\Delta E_{ \pm}$, are comparable to $E_{ \pm}$, the terms that ensue have no simple structure. All $(4 l+2)$ possible combinations will appear - with only $(l+2)$ independent parameters, of course, but that is of little help. Put another way, the relevant

${ }^{4}$ ) The two Kondo temperatures, $T_{\mathrm{K}}$ and $\tilde{T}_{\mathrm{K}}$, discussed above are not of that type : within VS symmetry, a Kondo cross over would lead to a non degenerate ground state, see section 4 . 
parameter space is an $(l+2)$ dimensional manifold in the more complete $(4 l+2)$ dimensional space allowed by symmetry. But that manifold is so twisted that one may as well ignore the corresponding restriction.

The situation is somewhat simpler when $\Delta E_{ \pm} \ll E_{ \pm}$. Then one can expand the energy denominators : the excited state splittings appear linearly, and a reasonably simple form of the Schrieffer Wolff interaction follows. However, one may still question the validity of the Hartree Fock approximation. Consequently, we prefer to rely only on symmetry, and to use all $(4 l+2)$ parameters that it allows.

Similar arguments apply in the presence of spin orbit coupling. Then the ground state of $H_{\mathrm{at}}^{0}$ corresponds to a well defined $J$, equal to

$$
L-S \text { if } n_{\mathrm{d}_{0}}<5, L+S \text { if } n_{\mathrm{d}_{0}}>5 .
$$

The coupling with the conduction electron must have the form

$$
(\mathbf{J} . \mathbf{s})_{\sigma \sigma^{\prime}}^{p} \cdot(\mathbf{J} . \mathbf{l})_{m m^{\prime}}^{q}
$$

The maximum number of independent coefficients is now

$$
2[2 \operatorname{Min}(J, l)+1] .
$$

In the presence of a crystal field, the situation is even more complicated. We shall only quote a few characteristic situations, in the case of a cubic field in transition metals (the $\mathrm{d}$ states split into an $\mathrm{E}$ doublet and $\mathrm{a} \mathrm{T}_{2}$ triplet).

Pure spin, $L=0$ case $\left(n_{\mathrm{d}_{0}}=5\right)$. - When projected on the cubic eigenstates, the effective Hamiltonian is diagonal

$$
\left[V_{\alpha} \delta_{\sigma \sigma^{\prime}}+\Gamma_{\alpha} \mathbf{S} \cdot \mathbf{s}_{\sigma \sigma^{\prime}}\right] C_{\alpha \sigma}^{*} C_{\alpha \sigma^{\prime}} .
$$

According to the Wigner Eckardt theorem, we have separate coefficients $V_{\mathrm{E}} \Gamma_{\mathrm{E}}, V_{\mathrm{T}} \Gamma_{\mathrm{T}}$ for the two representations. The difference between the " $\mathrm{E}$ » and « $\mathrm{T}$ » coefficients is due to crystal field splitting in the virtual ionized states : it should be very small.

Orbital singlet produced by the crystal field. Usually, the triplet one electron state lies below the doublet. Thus, from Hund's rule, the ground state for $n_{\mathrm{d}_{0}}=3$ is an orbital singlet with spin $3 / 2$ (the triplet is filled). Similarly, for $n_{\mathrm{d}_{0}}=8$ one has again an orbital singlet with spin 1 (two spin aligned holes in the doublet).

In such a singlet, only direct and spin exchange scattering may occur : the hamiltonian must again have the form (20), with four independent coefficients. The difference is that now the $\mathrm{E}$ and $\mathrm{T}$ channels have different orders of magnitude instead of being nearly similar. Consider for instance the $n_{\mathrm{d}_{0}}=3$ case. An « $\mathrm{E}$ » conduction electron can only hop into the impurity : it contributes to $T_{\alpha \alpha^{\prime}}^{+}$in (5); conversely, a « $\mathrm{T} »$ electron can only hop out : it contributes to
$T_{\alpha \alpha^{\prime}}^{-}$. In the latter case, the scattering electron senses directly the spin $S$ of the impurity : $\Gamma_{\mathrm{T}}$ is large. Instead, an impinging « $\mathrm{E} »$ electron can only sense $S$ indirectly, via configurational splittings in the virtual ionized state. $\Gamma_{\mathrm{E}}$ will thus be very small. The exchange anisotropy is thus extremely large : $\left|\Gamma_{\mathrm{T}}\right| \gg\left|\Gamma_{\mathrm{E}}\right|$ The reverse situation would hold for $n_{\mathrm{d}_{0}}=8$.

The $n_{\mathrm{d}_{0}}=1$ case. - The corresponding ground state of $H_{\text {at }}^{0}$ has symmetry $\mathrm{T}_{2}$, spin $1 / 2$. When coupled to an incoming conduction electron (either $\mathrm{E}$ or $\mathrm{T}_{2}$ ), the resulting orbital states split according to the scheme

$$
\begin{aligned}
T_{2} \otimes T_{2} & =A_{1}+E+T_{1}+T_{2}, \\
T_{2} \otimes E & =T_{1}+T_{2} .
\end{aligned}
$$

Each state is either a spin singlet or triplet. It follows that the scattering matrix $T_{\text {TT }}$ depends on 8 parameters : $V_{\mathrm{A}_{1}}, \Gamma_{\mathrm{A}_{1}}, \ldots, V_{\mathrm{T}_{2}}, \Gamma_{\mathrm{T}_{2}}$, while the matrix $T_{\mathrm{EE}}$ depends only on 4. Moreover, since the representations $\mathrm{T}_{1}, \mathrm{~T}_{2}$ appear in both products, a conversion scattering is also possible, with an extra 4 parameters. Altogether, 16 parameters, which is far too much ! Fortunately, many of them are very small. For instance, the spin exchange part of $T_{\mathrm{EE}}$ and $T_{\mathrm{ET}}$ arises again from $E_{ \pm}$splitting. We may neglect it : only $\Gamma_{\text {TT }}$ is important (scattering of an electron with the same symmetry as the impurity site). As regards T-T scattering, the VS symmetry holds in first approximation : out of the 8 corresponding parameters, only 2 are independent (the others arise from small departures from VS symmetry).

A similar analysis holds for $n_{\mathrm{d}_{0}}=9$ : the impurity has a single $\mathrm{E}$ hole which couples to an incoming electron according to the scheme

$$
\mathrm{E} \otimes \mathrm{T}_{2}=\mathrm{T}_{1}+\mathrm{T}_{2}, \quad \mathrm{E} \otimes \mathrm{E}=\mathrm{A}_{1}+\mathrm{A}_{2}+\mathrm{E} .
$$

Note that here there is no common representation in the two products : conversion scattering, $T_{\mathrm{ET}}$, is impossible. Here again, only the E-E spin exchange is important $\left({ }^{5}\right)$. That particular case has been discussed in some detail by Cragg et al. [13], who write second order scaling equations for the six coefficients of $\mathrm{E}-\mathrm{E}$ scattering.

In any given situation, a similar group theoretical analysis may be carried out - including if necessary spin orbit interaction. In the end, one must couple the ground state multiplet of $H_{\mathrm{at}}^{0}$, whatever it is, with either an $(\mathrm{E}, 1 / 2)$ or a $\left(\mathrm{T}_{2}, 1 / 2\right)$ conduction electron. The number of coefficients is usually large - and it is vital to establish a hierarchy of these coefficients, similar to that we just sketched. The approximate validity of VS symmetry is often of great use.

$\left({ }^{5}\right)$ Note that treating the $\mathrm{E}$ doublet as a fictitious spin $1 / 2$ (or the $\mathrm{T}_{2}$ triplet as a fictitious spin 1) would be incorrect. 
4. The low temperature strong coupling limit. 4.1 Stability OF THE FIXED POINT. - Consider a Kondo cross over in which the coupling strength increases from weak to strong. In the intermediate region, a perturbation expansion is of no avail. Unless one can proceed numerically [11], [14], we must rely on reasonable guesses.

The first question is to identify the strong coupling limit. It is usually easy to find the corresponding ground state : we just need to severe the hopping of conduction electrons from the impurity site to the rest of the crystal. We are left with a local problem involving the impurity itself, and an arbitrary number of trapped conduction electrons. The impurity is described by $H_{\mathrm{at}}^{0}$ - or by its projection onto its ground state at low temperature; the number of trapped electrons (leading to an effective valency) is a priori unknown, anywhere between 0 and $2(2 l+1)$. The number of such eigenstates is finite : finding the lowest one is an easy task $\left(^{6}\right)$.

Two possibilities may then occur : that new ground state is either degenerate or non degenerate. If it is degenerate, treating hopping between the impurity site and the rest of the world within second order perturbation theory leads to a new effective scattering interaction; conduction electrons on the first layer of neighbours scatter off the impurity site, thereby changing its state within the new ground multiplet. The new scattering amplitude is of order

$$
U \sim \frac{D^{2}}{T}
$$

The strong coupling Kondo problem for the bare impurity $(T \rightarrow \infty)$ turns into a weak coupling Kondo problem $(U \rightarrow 0)$ for the dressed impurity (frozen with its trapped electrons). It is usually straightforward to study the stability of the $U=0$ fixed point - again there are two possibilities.

- If that fixed point is stable, a Kondo cross over can proceed to $T \rightarrow \infty$. Of course, we do not prove it does, but at least a scaling trajectory going from $T=0$ to $T=\infty$ is sensible.

- If the $U=0(T=\infty)$ fixed point is unstable, then the trajectory must go somewhere else, either at an intermediate coupling fixed point, or in another direction of parameter space (for instance via the growth of initially small anisotropies).

We shall meet examples of both possibilities later.

${ }^{6}$ ) One may worry that the interaction between conduction electrons, that has been ignored in our model, might affect deeply the choice of that effective valency. Actually, one must remember that our scaling procedure implies an ever increasing length unit. By the time we reach $T_{\mathrm{K}}$, the conduction electron cloud is so spread out that interactions between conduction electrons are negligible. This is a physical way to state that two particle interactions in the conduction band are not relevant in the sense of scaling.
If the new ground state is non degenerate, there is no Kondo effect left : everything is analytical as $D \rightarrow 0$. Then the strong coupling fixed point is stable, and its approach can be described in terms of the Landau theory picture devised by one of us [6].

Usually, the diverging scattering amplitude $T$ depends on several parameters (only in the elementary Kondo problem does a single $\Gamma$ become infinite). Consequently, strong coupling is somewhat ambiguous. It corresponds to a multidimensional space, in which several components may go to $\infty$ independently. The nature of the new ground state (and the effective valency) depends on the ratios of these parameters - i.e. on the direction of the trajectory in parameter space. There will exist ranges of asymptotic directions that correspond to a given ground state. Choosing unambiguously between these cones in parameter space would mean that we can follow the trajectory througkout the intermediate cross over region. This we cannot do : thus, we must rely on extrapolations of the first steps of scaling. Such arguments are again reasonable guesses, that may be convincing, but never rigorous : the choice of the ground state - and thus the $T \rightarrow 0$ physics - is often subject to some ambiguity.

In practice, all examples worked out today eventually evolve towards non degenerate ground states, except if some extra unphysical symmetries are imposed on the problem. It is not clear to us whether that conclusion follows from general analyticity and continuity arguments, as claimed by Anderson.

4.2 The FERMI LIQUID PICTURE OF NON DEGENERATE GROUND STATES. - It is based on a simple physical idea : virtual hopping to and from the impurity site (i) modifies the conduction electron self energy, (ii) generates an indirect interaction between conduction electrons, via the polarizability of the frozen impurity. The effective Hamiltonian is retarded (it is actually a self energy, function of an energy $z$ which we measure from the Fermi level). It can only involve the local combination $C_{m \sigma}$ of conduction electron operators, summed over all $k$ (the interaction was local at the beginning : it remains so at the end). When the cut off (i.e. the temperature) goes to zero, we need keep only the leading terms in powers of $D$ :

- a one body potential up to first order in $z$,

- a two body interaction to zeroth order in $z$.

Further powers in $z$, or interactions between more than two electrons do exist, but they are of higher order in $D$ and thus irrelevant in the approach to the fixed point. The philosophy of that approach is discussed in some detail in ref. [7].

In the usual $l=0, s=1 / 2$ case, the effective Hamiltonian (invariant under spin rotation) is simply

$H_{\text {eff }}=\left(V_{0}+V_{1} z\right) C_{\sigma}^{*} C_{\sigma}+U C_{\uparrow}^{*} C_{\downarrow}^{*} C_{\downarrow} C_{\uparrow}$

$V_{0}$ characterizes the fixed point itself $\left(\sim D^{0}\right), V_{1}$ and $U$ 
the approach to the fixed point $(\sim D)$. Note that there is only one interaction parameter. (The exclusion principle precludes two particles with the same spin on the same site : they cannot interact.) From (21), we infer a similar expansion for the phase shift, which is a function of the energy $z$, the spin $\sigma$ of the colliding particle and the distribution of other particles. Assuming a spin polarization along the $z$-axis $\left({ }^{7}\right)$, we may write the phase shift as an expansion :

$$
\delta_{\sigma}\left(z, \delta n_{\sigma^{\prime}}\right)=\delta_{0}+\alpha z+\varphi \delta n_{-\sigma}+\cdots
$$

(where $\delta n_{\sigma}$ is the departure from the ground state distribution). From $\delta$, one infers the scattering state energy, hence the specific heat and the spin susceptibility. Moreover, $\delta$ yields the resistivity right away [6]. The low temperature physics thus depends on three parameters only : $\delta_{0}, \alpha$ and $\varphi$.

Let us now turn to the orbital case, in the absence of crystal field or spin orbit effects. The effective Hamiltonian must be invariant separately under orbital and spin rotations. (21) is thus replaced by

$$
\begin{aligned}
H_{\mathrm{eff}}=\left(V_{0}+V_{1} z\right) C_{m \sigma}^{*} C_{m \sigma}+U\left(m_{\mathrm{i}} \sigma_{\mathrm{i}}\right) \times \\
\\
\times C_{m_{1} \sigma_{1}}^{*} C_{m_{2} \sigma_{2}}^{*} C_{m_{3} \sigma_{3}} C_{m_{4} \sigma_{4}}
\end{aligned}
$$

$U$ describes the interaction of two electrons (because of phase space) in the same energy shell : it can depend only on $(2 l+1)$ parameters, corresponding to the $(2 l+1)$ states one can form with two electrons :

$$
\begin{array}{llrl}
L & =0,2, \ldots, 2 l \quad ; & S & =0 \\
L & =1,3, \ldots, 2 l-1 ; & S & =1 .
\end{array}
$$

(There are only half as many parameters as in the Schrieffer Wolff Hamiltonian because here the electrons are indistinguishable.)

From (23), one may construct an expansion of the phase shift matrix, $\delta_{\substack{m \sigma \\ \boldsymbol{m}^{\prime}}}$, which again is a functional of the particle distribution $\delta n_{\substack{m \sigma \\ m^{\prime} \sigma^{\prime}}}$. In analogy with (22), that expansion will involve

- the Fermi level phase shift $\delta_{0}$,

- a parameter $\alpha$ which provides the energy scale $\left(T_{\mathrm{K}}\right)$,

- $(2 l+1)$ interaction parameters.

From $\delta$, we infer all physical quantities by a straightforward generalization of [7].

These $(2 l+1)$ parameters are actually a maximum. We shall see that symmetry and universality arguments reduce that number considerably. In the presence of spin orbit or crystal field effects, a similar group theoretical analysis would yield even more parameters.

$\left({ }^{7}\right)$ For an arbitrary spin polarization, the phase shift is a spin matrix, which would complicate the writing but not the physics the spin of the colliding particle may precess around the local magnetization in the course of scattering.
4.3 TO WHAT EXTENT DOES ONE EXPECT UNIVERSAL RESUltS AS $T$ GOES TO ZERO. - The less independent parameters in the $T \rightarrow 0$ limit, the more universal the behaviour. We here survey the various arguments that can help eliminating as many parameters as possible from the above maximum number.

The first type of argument relies on symmetry. The rotational symmetry is already built in. In addition, after a given cross over, new symmetries may appear. For instance, in the case $L=0$, the effective Hamiltonian (18) conserves $m$, a symmetry which did not exist before the excited states of $\varepsilon_{0}$ decoupled. Clearly that symmetry exists only if the intermediate range is broad enough. One must find a region where $D$ is still $\gg T_{\mathbf{K}}$ (the Kondo cross over did not start), and nevertheless small enough that the new symmetry is well established. That implies of course $D \ll E_{ \pm}$ (Schrieffer Wolff transformation valid), but also $D<\Delta E_{0}$ (decoupling of excited states in $\varepsilon_{0}$ completed). If that condition $T_{\mathrm{K}} \ll \Delta E_{0}$ is met, the symmetry that was established in the intermediate range will be preserved throughout scaling down to $T=0$ : hence a considerable reduction in the number of parameters.

Such symmetries become exact when $T_{\mathrm{K}} \rightarrow 0$. Otherwise, they are only approximate, with errors $\sim T_{\mathrm{K}} / \Delta E_{0}$.

The second type of argument relies on universality. The Kondo cross over represents a singularity of width $T_{\mathrm{K}}$ attached to the Fermi level $\mu$. If $T_{\mathrm{K}}$ is much smaller than all other energy scales in the problem (the original cut off $D_{0}$, splittings $E_{ \pm}$or $\Delta E_{0}$ ), that singularity must move rigidly with $\mu$. Put another way, if one moves $\mu$ and the energy $z$ by the same amount, the phase shift $\delta$ must be invariant (moving $z$ and $\mu$ up by a small amount $\varepsilon \sim T_{\mathrm{K}}$ is tantamount to moving all other energy levels by $-\varepsilon$, which should make little difference if $\varepsilon$ is $\left.\ll \Delta E_{0}, \ldots\right)$. That universality requirement imposes one extra condition on the interaction parameters. (In the usual s-d model, it fixes the single parameter $U$ and yields the famous Wilson result $\chi T / C_{\mathrm{v}}=2$.) Once again, that universality condition is correct to order $T_{\mathrm{K}} / \Delta E$, whatever splitting is considered.

A stronger universality holds if the scattering channels $\alpha$ split into several classes that are not coupled by the scattering matrix $T_{\alpha \alpha^{\prime}}$ (e.g. the different $m$ channels in the above $L=0$ case). Then an electron in one class never jumps into another class. Let us assign independent Fermi levels $\mu_{\alpha}$ to each channel (which is equivalent to an orbital and spin magnetic field). An electron in channel $\alpha$ belonging to one class cannot sense directly the Fermi level $\mu_{\alpha^{\prime}}$ in other classes. Thus the corresponding phase shift $\delta_{\alpha}$ should be independent of $\mu_{\alpha^{\prime}}$. Actually, there remains a weak indirect dependence via the impurity dynamics. But the corresponding correction is $\sim \delta \mu_{\alpha^{\prime}} / \Delta E_{0}$, very small. A large correction $\sim \delta \mu_{\alpha^{\prime}} / T_{\mathrm{K}}$ would arise only if an electron within an energy range $T_{\mathrm{K}}$ could 
jump from one channel to the other, carrying into $\alpha^{\prime}$ information about the Kondo singularity in $\alpha$.

Such a stronger universality will impose further requirements on the low temperature coefficients with once more an accuracy $\sim T_{\mathrm{K}} / \Delta E_{0}$ (due to the above self energy corrections).

All the above criteria were related to the smallness of $T_{\mathrm{K}}$. In addition, there may exist weaker conditions which are related to the structure of the initial Hamiltonian. A typical example is approximate VS symmetry in the case $n_{\mathrm{d}_{0}}=1$, with an accuracy $\sim \Delta E_{ \pm} / E_{ \pm}$. That symmetry will be reflected in the low temperature behaviour. A similar situation holds for crystal field effects, which may lead to $\Gamma_{\mathrm{E}} \gg \Gamma_{\mathrm{T}}$ (or the reverse). Such arguments may help in reducing the number of parameters in the Landau theory picture. We should however insert one caveat : we implicitly assume that small departures from the approximate symmetry remain small throughout scaling. Although likely, this is not necessarily true (see for instance the discussion of (17) where a correction $C_{\lambda}$ may become very large if the exponent $S_{\lambda} / M>1$ ).

One final remark : in any cross over, the trajectory is perfectly well defined if we know its starting point. For instance, assume that we start from VS symmetry, and project it on the ground state multiplet $L S$ (the second order scaling corrections being still negligible when $D \sim \Delta E_{0}$ ). VS symmetry is no longer preserved by further scaling, but the corresponding trajectory is uniquely determined. In that sense, we may say that the low temperature behaviour is universal, to the extent that it depends only on one parameter (all the 5 interaction coefficients in (23) have prescribed values). However that remark is of little help if we cannot calculate those values. The weak universality requirement provides one condition, but we have no further symmetry argument to help. Only a numerical calculation $a$ la Wilson can provide the (unambiguous) result.

If the Kondo cross over is already started by the time $D \sim \Delta E_{0}$, the low temperature behaviour will depend on the ratio $T_{\mathrm{K}} / \Delta E_{0}$ : the behaviour is less and less universal as there are more atomic splittings of $H_{\mathrm{at}}^{0}$ in the cross over region. A theoretical analysis of the intermediate regime is very hard : it may be wiser to interpolate between the two limiting cases $T_{\mathrm{K}} \ll \Delta E_{0}$ and $T_{\mathrm{K}} \gg \Delta E_{0}$.

5. The orbital singlet case. - We assume that the ground state of $H_{\mathrm{at}}^{0}$ is an orbital singlet. In the absence of anisotropies, the Schrieffer Wolff hamiltonian has the form (18). The kinematics depends only on two parameters

- the spin $S$ of the impurity,

- the number $n$ of orbital channels, here $(2 l+1)$.

For transition metal impurities, such an isotropic orbital singlet obtains only for $n_{\mathrm{d}_{0}}=5$ (i.e. $\mathrm{Mn}$ in $\mathrm{Cu})$. Then

$$
n=2 S \text {. }
$$

We shall nevertheless, as a first try, consider $n$ and $S$ as independent parameters. This is not completely stupid if we think of crystal field effects. In the case $n_{\mathrm{d}_{0}}=5$, we may imagine extreme anisotropy, $\Gamma_{\mathrm{T}} \gg \Gamma_{\mathrm{E}}$. Then the E channels are irrelevant, and in practice

$$
n=3<2 S \text {. }
$$

Conversely, we saw that crystal field splittings produced singlet ground states for $n_{\mathrm{d}_{0}}=3$ and 8. If we ignore anisotropies $\left(\Gamma_{\mathrm{T}}=\Gamma_{\mathrm{E}}\right)$, then we have

$$
n=5>2 S \text {. }
$$

Actually, such requirements on anisotropy are just the opposite of what happens : from the previous discussion, we expect a small anisotropy of $\Gamma$ if $n_{\mathrm{d}_{0}}=5$, a large one if $n_{\mathrm{d}_{0}}=3$ or 8 . Nevertheless, we shall first discuss the behaviour of the isotropic pure spin Kondo problem with arbitrary values of $n$ and $S$. Besides its academic interest (it provides simple examples of the various behaviours sketched in section 2), it is the starting point for a study of anisotropy effects.

5.1 STRONG COUPLING FIXED POINTS FOR AN ISOTROPIC ORBITAL SINGLET. - The hamiltonian (18) depends on two dynamical parameters, $V$ and $\Gamma$. The scalar potential is not logarithmically relevant. In lowest order, it does not change upon scaling. More generally, its value follows from simple electron-hole symmetry considerations (after an appropriate change of basis : see Cragg and Lloyd [15] and ref. [7] for more details). The strong coupling limit corresponds to $\Gamma \rightarrow+\infty$. Then the impurity traps as many electrons as possible - i.e. one per orbital channel - with spin opposite to $S$. The resulting complex has $\operatorname{spin} S-\frac{n}{2}$. Hence three possibilities

(i) $n=2 S$ : The ground state is non degenerate; the strong coupling fixed point is attractive and the usual Fermi liquid picture applies. This is just the case of $\mathrm{Mn}$ in $\mathrm{Cu}$. If we return to the original Anderson model, the ground state is obtained by forming singlet combination of one impurity electron $\left(a_{m \sigma}\right)$ and one conduction electron $\left(C_{m \sigma}\right)$ in each orbital channel. Clearly, there is only one way to do so.

(ii) $2 S>n$ : The trapped electrons cannot quench completely the spin $S$. The dressed impurity remains magnetic with spin $S^{\prime}=S-n / 2$. A typical configuration with maximum $S_{z}^{\prime}$ is shown on figure $2 a$ : the impurity spin $S$ is up, and all the trapped electrons are down in order to maximize the exchange energy. Virtual hopping to the first layer of neighbours (with strength $D$ ) generates a new Kondo effect between $S^{\prime}$ and the conduction electrons, with a weak coupling $\Gamma^{\prime} \sim D^{2} / \Gamma$. The central point is now that such a coupling is ferromagnetic. In order to prove it, consider the arrangement of figure $2 a$. A nearby electron with down spin cannot jump onto the central site because of the exclusion principle (all available states are 


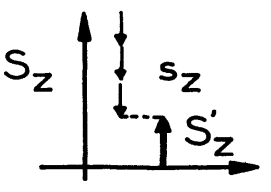

(a)

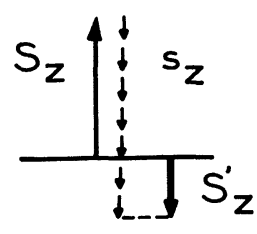

(b)
Fig. 2. - The strong coupling arrangement of spins for the impurity and $n$ trapped electrons (a) when $n<2 S$, (b) when $n>2 S$.

already occupied). Its energy is thus unchanged. On the contrary an up spin electron jumps in freely : its energy is lowered according to standard second order perturbation theory. Altogether, a nearby electron has a lower energy if its spin is parallel to $S_{z}^{\prime}$ : the coupling is indeed ferromagnetic !

The strong $\Gamma$ coupling is equivalent to a weak ferromagnetic $\Gamma^{\prime}$ limit : we know that the latter fixed point is attractive. We conclude that the $\Gamma \rightarrow \infty$ fixed point is stable. A simple scaling trajectory going from $\Gamma=0$ to $\Gamma=\infty$ as the cut off goes to zero is thus possible. We do not prove that the cross over occurs, but at least there is no contradiction - and usually the simplest guess is the good one ! Indeed, numerical calculations by Cragg and Lloyd [15] in the case $n=1, S>1 / 2$ have shown that the system evolved toward an incompletely quenched impurity spin : the strong coupling fixed point is indeed reached. In their remarkable piece of work, they show that the approach to that fixed point is still logarithmic (as it should for an effective Kondo effect with spin $\mathbf{S}^{\prime}$ ), in contrast to the case $n=2 S$ where the approach is analytic.

(iii) $2 S<n$ : The corresponding strong coupling arrangement is pictured on figure $2 b$. There again, a nearby electron with up spin has its energy lowered by second order perturbation corrections, while an up spin electron is unaffected. The difference is that $S_{z}^{\prime}$ is now opposite to $S_{z}$. The effective Kondo coupling $\Gamma^{\prime}$ between $S^{\prime}$ and the conduction band is thus antiferromagnetic. We know that the corresponding weak coupling fixed point is repulsive. We conclude that the $\Gamma \rightarrow \infty$ fixed point is unstable. The scaling trajectory, sandwiched between two unstable fixed points, has the shape shown on figure $3 b$ : there must necessarily exist at least one fixed point with finite $\Gamma$ in between.

One might imagine other situations as $D \rightarrow 0$, for instance trapping only $2 S$ electrons in order to make a spin singlet. But then the resulting state would have a net orbital angular momentum on the impurity site, and an effective orbital Kondo effect would ensue : it is easily seen that the corresponding fixed point is also repulsive. Anyhow, that would break the original $m$-symmetry of the Hamiltonian (18), which is forbidden. Thus, we cannot avoid the fixed point at finite $\Gamma$, of the type discussed by Abrikosov
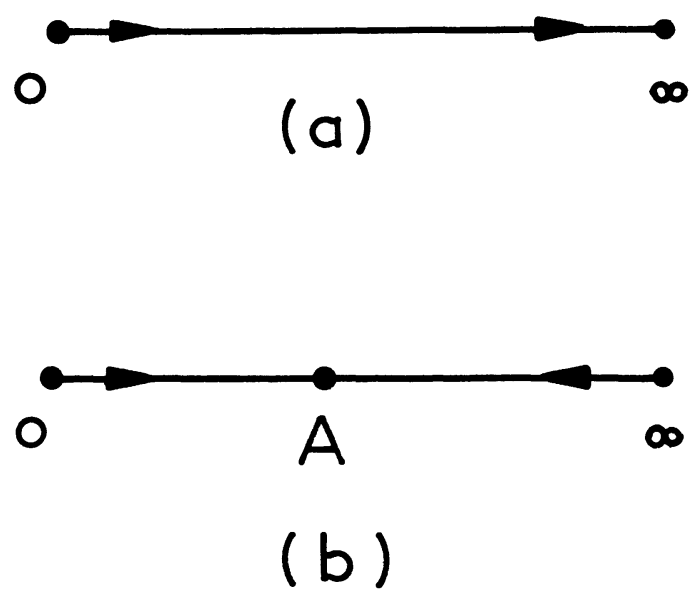

Fig. 3. - The scaling trajectories along the $\Gamma$ axis, (a) when $n<2 S$, (b) when $n>2 S$ : an extra fixed point A with finite $\Gamma$ must exist somewhere.

and Migdal [16], Fowler and Zawadowski [17] and others.

Such a conclusion is comforted by the limit $n$ very large, in which we can prove the existence of that fixed point. Let us return to the weak coupling perturbation expansion of the scaling equation (10). Each term in the expansion may be represented by a diagram. The second order diagrams are shown on figure 1 ; the third and fourth order ones are shown on figure 4 . Since the basic scattering vertex (18) con-
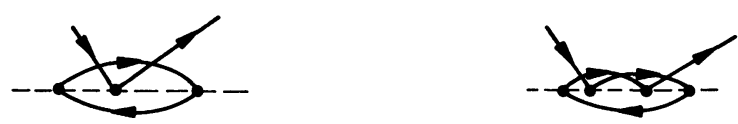

Fig. 4. - The third and fourth order diagrams in the scaling equation for $\Gamma$.

serves $m$, the orbital quantum number is conserved along each full electron line in the diagrams. The only way in which the number of channels enters is in an extra factor $n$ for each electron closed loop (which may belong to any channel). The scaling equation is thus replaced by

$\frac{\mathrm{d} \Gamma}{\mathrm{d} \log D}=-\rho \Gamma^{2}+n \rho^{2} \Gamma^{3}+c \rho^{3} \Gamma^{4}+\cdots$

( $c$ depends on $S$, but not on $n$ : it is given in [16]). If we retain only the first two terms in (25), we find a fixed point at

$$
\rho \Gamma^{*}=\frac{1}{n}
$$

When $n=1$, that result is meaningless, since the expansion does not converge. On the other hand, if $n$ is large, $\Gamma^{*}$ is small and the result is meaningful. Every additional vertex yields a factor $\Gamma^{*}=1 / n$, every additional closed loop yields a factor

$$
n \Gamma^{* 2}=1 / n
$$


Both corrections are small : we have a bona fide perturbation expansion, and retaining only the first two terms of (25) is justified. In the limit $n \gg 1$, we have thus proved the existence of $\Gamma^{*}$. According to our stability discussion, such a fixed point must persist as long as $n>2 S$.

The final proof belongs to numerical calculation. Once again Cragg and Lloyd [13] have shown the existence of $\Gamma^{*}$ by adapting the approach of Wilson to our more complicated multichannel model.

5.2 INFLUENCE OF ANISOTROPY : RETURN TO NORMAL BEHAVIOUR. - In practice the above fixed point $\Gamma^{*}$ is not physically attainable, as it is unstable with respect to anisotropies. Consider for instance cubic anisotropy for $l=2: \Gamma$ splits into two couplings $\Gamma_{\mathrm{E}}$ and $\Gamma_{\mathrm{T}}$ for incoming electrons with symmetry $\mathrm{E}$ or $\mathrm{T}_{2}$. The scaling expansion (25) is replaced by

$$
\begin{aligned}
& \frac{\mathrm{d} \Gamma_{\mathrm{E}}}{\mathrm{d} \log D}=-\Gamma_{\mathrm{E}}^{2}+\Gamma_{\mathrm{E}}\left(3 \Gamma_{\mathrm{T}}^{2}+2 \Gamma_{\mathrm{E}}^{2}\right)+\cdots \\
& \frac{\mathrm{d} \Gamma_{\mathrm{T}}}{\mathrm{d} \log D}=-\Gamma_{\mathrm{T}}^{2}+\Gamma_{\mathrm{T}}\left(3 \Gamma_{\mathrm{T}}^{2}+2 \Gamma_{\mathrm{E}}^{2}\right)+\cdots .
\end{aligned}
$$

(For simplicity, we have set $\rho=1$ by an appropriate choice of units.) If we stop the expansion there, we find a fixed point at $\Gamma_{\mathrm{E}}^{*}=\Gamma_{\mathrm{T}}^{*}=\frac{1}{5}$. In the vicinity of that fixed point we set

$$
\Gamma_{\mathrm{E}}-1 / 5=\gamma_{\mathrm{E}}, \quad \Gamma_{\mathrm{T}}-1 / 5=\gamma_{\mathrm{T}} .
$$

The linearized scaling equations read

$$
\begin{aligned}
& \frac{\mathrm{d} \gamma_{\mathrm{E}}}{\mathrm{d} \log D}=-\frac{\gamma_{\mathrm{E}}}{25}+\frac{6 \gamma_{\mathrm{T}}}{25} \\
& \frac{\mathrm{d} \gamma_{\mathrm{T}}}{\mathrm{d} \log D}=\frac{4 \gamma_{\mathrm{E}}}{25}+\frac{\gamma_{\mathrm{T}}}{25} .
\end{aligned}
$$

The corresponding eigenvalues and eigenvectors are

$$
\begin{aligned}
\gamma_{\mathrm{E}}=\gamma_{\mathrm{T}}=\gamma, & \mathrm{d} \gamma / \mathrm{d} \log D=\gamma / 5 \\
\begin{cases}\gamma_{\mathrm{E}}=3 \gamma \\
\gamma_{\mathrm{T}}=-2 \gamma,\end{cases} & \mathrm{d} \gamma / \mathrm{d} \log D=-\gamma / 5 .
\end{aligned}
$$

As expected the fixed point $\Gamma^{*}$ is stable if approached along the diagonal in the $\left(\Gamma_{\mathrm{E}}, \Gamma_{\mathrm{T}}\right)$ plane $\left(\gamma \sim D^{1 / 5}\right)$. On the other hand, it is unstable along the separatrix $3 \gamma_{\mathrm{T}}+2 \gamma_{\mathrm{E}}=0\left(\gamma \sim D^{-1 / 5}\right) . \Gamma^{*}$ is a saddle point, and anisotropies grow as $D \rightarrow 0$, even if they were initially small. That conclusion relies on the expansion (27) : it should remain qualitatively true in higher orders.

In order to sketch the trajectories in the $\Gamma_{\mathrm{E}}, \Gamma_{\mathrm{T}}$ plane, we first note that scaling preserves the relations $\Gamma_{\mathrm{E}}=0, \Gamma_{\mathrm{T}}=0, \Gamma_{\mathrm{E}}=\Gamma_{\mathrm{T}}$. The axes and diagonal are special trajectories we cannot cross. We next identify the fixed points and their stability. Near the unstable origin, we have

$$
\frac{\mathrm{d} \Gamma_{\mathrm{E}}}{\mathrm{d} \Gamma_{\mathrm{T}}}=\frac{\Gamma_{\mathrm{E}}^{2}}{\Gamma_{\mathrm{T}}^{2}}
$$

whose solution is

$$
\Gamma_{\mathrm{T}}=\frac{\Gamma_{\mathrm{E}}}{1+a \Gamma_{\mathrm{E}}}
$$

with $a$ an arbitrary constant : trajectories are tangent to the diagonal. In the strong coupling limit, the system is equivalent to a weak antiferromagnetic Kondo coupling with the quenched spin, with strength

$$
\Gamma_{\mathrm{E}}^{\prime} \sim \frac{D^{2}}{\Gamma_{\mathrm{E}}}, \quad \Gamma_{\mathrm{T}}^{\prime} \sim \frac{D^{2}}{\Gamma_{\mathrm{T}}}
$$

(virtual hopping of an $\alpha$-electron increases the impurity site energy by $\Gamma_{\alpha}$ ). Thus the scaling equation (28) for $\Gamma^{\prime}$ turns into the following relation for $\Gamma$

$$
\Gamma_{\mathrm{T}}=\Gamma_{\mathrm{E}}+a .
$$

Scaling trajectories are parallel to the diagonal at $\infty$.

Further information will depend on the spin $S$. Consider first $S=1\left(n_{\mathrm{d}_{0}}=8\right) . \Gamma_{\mathrm{E}}=\infty, \Gamma_{\mathrm{T}}=0$ is a stable fixed point : the impurity spin is quenched by two $\mathrm{E}$ electrons, and the $\mathrm{T}$ channel disappears altogether. Along the $\Gamma_{\mathrm{T}}$ axis, we have a three channels, $S=1$ situation. According to our general discussion, the fixed point $\Gamma_{\mathrm{T}}=\infty, \Gamma_{\mathrm{E}}=0$ is unstable along the $\Gamma_{\mathrm{T}}$ axis, and there must exist a stable fixed point $\Gamma_{\mathrm{T}}^{*}$ somewhere on that axis. Note that the fixed point at $\infty$ is stable for small $\Gamma_{\mathrm{E}}$ couplings : the effective quenched spin $\mathbf{S}^{\prime}$ is reversed as compared to $\mathbf{S}$, and the original weak antiferromagnetic $\Gamma_{\mathrm{E}}$ turns into a weak ferromagnetic $\Gamma_{\mathrm{E}}^{\prime}$, which is attractive. Putting all these informations together, we may draw the scaling pattern of figure 5 , the simplest one consistent with known limits.

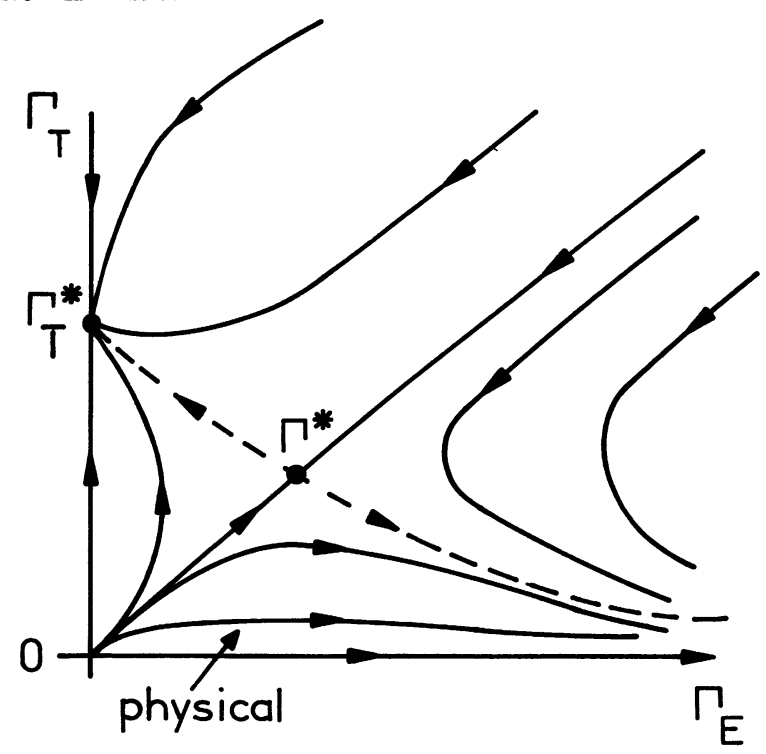

Fig. 5. - A sketch of the scaling trajectories for a spin $S=1$ impurity with cubic anisotropy $(l=2)$. The dashed line is the separatrix. A typical physical trajectory is marked with an arrow. 
A similar analysis holds for $S=3 / 2\left(n_{\mathrm{do}_{\mathrm{o}}}=3\right)$. $\Gamma_{\mathrm{T}}=\infty, \Gamma_{\mathrm{E}}=0$ is a stable non magnetic fixed point. If $\Gamma_{\mathrm{E}}=\infty, \Gamma_{\mathrm{T}}=0$, the impurity spin is incompletely quenched ( $S=3 / 2$, two channels) : the fixed point is attractive along the $\Gamma_{\mathrm{E}}$ axis, repulsive along the other direction (the weak $\Gamma_{\mathrm{E}}$ does not change its sign). More generally, for infinite $\Gamma_{\mathrm{E}}$, finite $\Gamma_{\mathrm{T}}$, we have an effective spin $1 / 2$, three channel situation : there must exist a fixed point at some finite $\Gamma_{\mathrm{T}}^{*}$. Thus we expect the scaling pattern shown on figure 6 (assuming $\Gamma_{\mathrm{T}}^{*}<\Gamma^{*}$, which looks reasonable in view of (26) : the relative excess of channels is larger for $\Gamma_{\mathrm{T}}^{*}$ ).

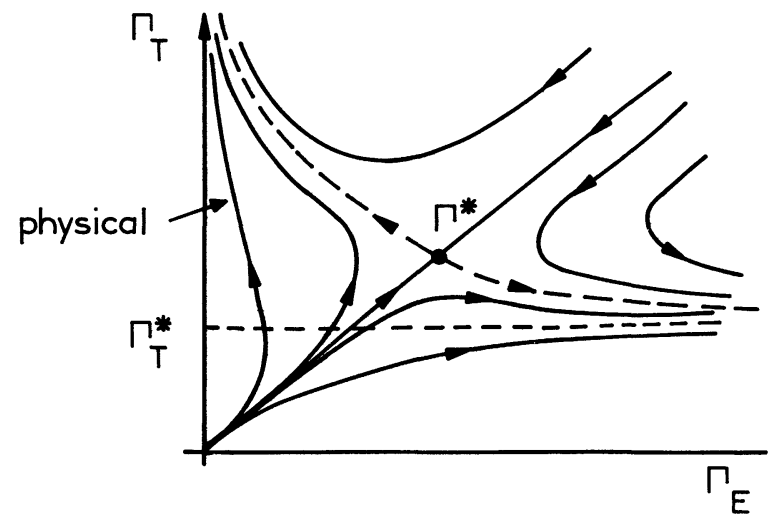

Fig. 6. - A sketch of the scaling trajectories for a spin $S=3 / 2$ impurity with cubic anisotropy $(l=2)$. The dashed line is the separatrix. A typical physical trajectory is marked with an arrow.

In practice the above analysis is academic. We have seen that for $S=1$ we expected $\Gamma_{\mathrm{E}} \gg \Gamma_{\mathrm{T}}$, while for $S=3 / 2$ we should have $\Gamma_{\mathrm{T}} \gg \Gamma_{\mathrm{E}}$. The corresponding trajectories are marked with an arrow on figures 5 and 6 : they go to the rather trivial non magnetic fixed point as $D \rightarrow 0$, in accordance with the general arguments of Anderson. The other exotic parts of the scaling diagrams cannot be reached physically, at least in the simple examples considered. (They are nevertheless of pedagogical interest, showing how one may tackle a difficult problem by an encircling strategy without resorting to numerical calculations.) The general conclusion that emerges is that anisotropies amplify throughout scaling, the "minority channels » being progressively eliminated by the quenching of $S$. The low temperature behaviour is regular (if for some unknown reason the initial conditions could lead to a non trivial fixed point, physical properties would display non analytic behaviour : see [16]).

To complete our survey of anisotropy effects, we return to the case $S=5 / 2$ (i.e. $n=2 S$ ). The strong coupling fixed point is non magnetic for arbitrary ratio $\Gamma_{\mathrm{E}} / \Gamma_{\mathrm{T}}$ : we expect a line of fixed points at infinity. The scaling trajectories should behave as shown on figure 7 : the anisotropy after the Kondo cross over reflects the one that existed before. Such a $D \rightarrow 0$ anisotropy is physically important, as it will control the phenomenological parameters of the Fermi liquid description (the lack of symmetry being reflected in the

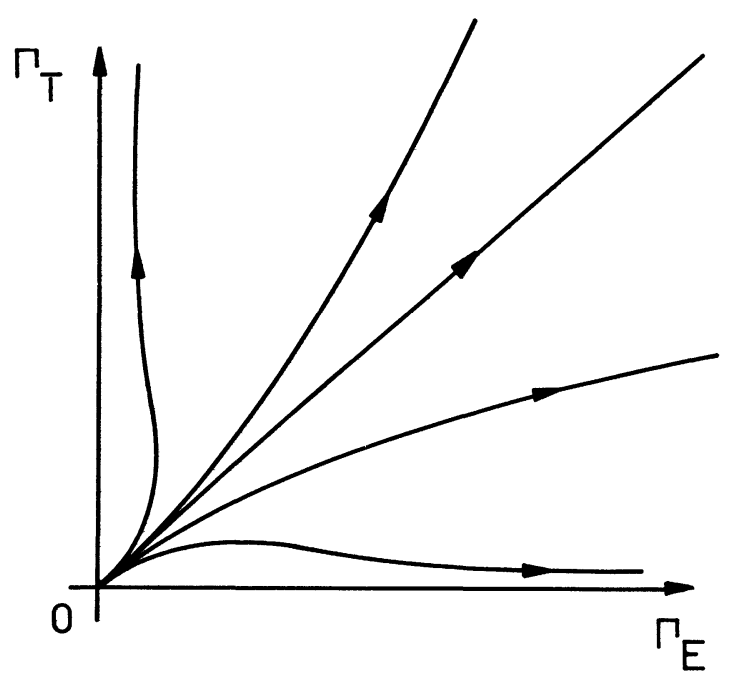

Fig. 7. - A sketch of the scaling trajectories for spin $S=5 / 2$ impurity with cubic anisotropy $(l=2)$.

interaction terms). For large $T_{\mathrm{K}}$, the initial $\Gamma$ is large : the final anisotropy is comparable to the initial one. If on the contrary $T_{\mathrm{K}}$ is very small, we start from a very weak coupling for which the trajectories are tangent to the diagonal. It follows that an initially small anisotropy $\left(\Gamma_{\mathrm{E}} / \Gamma_{\mathrm{T}} \approx 1\right)$ will rapidly become very large, the larger $\Gamma$ killing the other one. Such a growth of anisotropies is a weak coupling effect, which we can describe with the help of (27). From the relationship

$$
\frac{\mathrm{d} \Gamma_{\mathrm{E}}}{\mathrm{d} \Gamma_{\mathrm{T}}}=\frac{\Gamma_{\mathrm{E}}^{2}-\Gamma_{\mathrm{E}}\left(3 \Gamma_{\mathrm{T}}^{2}+2 \Gamma_{\mathrm{E}}^{2}\right)}{\Gamma_{\mathrm{T}}^{2}-\Gamma_{\mathrm{T}}\left(3 \Gamma_{\mathrm{T}}^{2}+2 \Gamma_{\mathrm{E}}^{2}\right)}
$$

we infer the scaling equation for the ratio $x=\Gamma_{\mathrm{E}} / \Gamma_{\mathrm{T}}$

$$
\frac{\mathrm{d} x}{\mathrm{~d} \Gamma_{\mathrm{T}}}=\frac{1}{\Gamma_{\mathrm{T}}} \frac{x^{2}-x}{1-\Gamma_{\mathrm{T}}\left(2 x^{2}+3\right)} .
$$

We see at once that the isotropic limit $x=1$ is unstable. Consider for instance the case $x<1$ (in the reverse situation, we would take $\Gamma_{\mathrm{E}}$ as a variable instead of $\Gamma_{\mathrm{T}}$ ). Eq. (27) only makes sense if $\Gamma_{\mathrm{T}} \ll 1$ : then we can ignore the denominator in (30); the solution of that differential equation is straightforward :

$$
x=\frac{x_{0}}{x_{0}+\left(1-x_{0}\right) \Gamma_{\mathrm{T}} / \Gamma_{\mathrm{T}_{0}}} .
$$

If we start from a very small $\Gamma_{\mathrm{T}_{0}} \ll 1, x$ is $\ll 1$ by the time we reach the Kondo cross over $\Gamma_{\mathrm{T}} \sim 1$, even if $x_{0} \sim 1$. Quite generally, (31) provides an estimate of the final anisotropy in terms of the initial one. A similar result holds for $x\left(\Gamma_{\mathrm{E}}\right)$ if $x>1$. That final anisotropy will be directly reflected in the Landau parameters at $T<T_{\mathrm{K}}$, and thus it will control the amount of universality of the low temperature behaviour.

5.3 Fermi LiQuid Parameters AND Universality. - We first consider the isotropic system characterized 
by the Schrieffer Wolff hamiltonian (18) : $2 l+1$ orbital channels, equivalent and completely decoupled (except indirectly via the dynamics of $\mathbf{S}$ ). Each vertex conserves $m$ : thus in any diagram, each conduction electron line corresponds to a specific $m$, the same as the line keeps interacting with the impurity spin. It follows that the basic electron-electron vertex (responsible for the interaction term in (23)) has the structure shown on figure $8:$ it contains only terms

$$
C_{m \sigma_{1}}^{*} C_{m^{\prime} \sigma_{2}}^{*} C_{m^{\prime} \sigma_{3}} C_{m \sigma_{4}}
$$

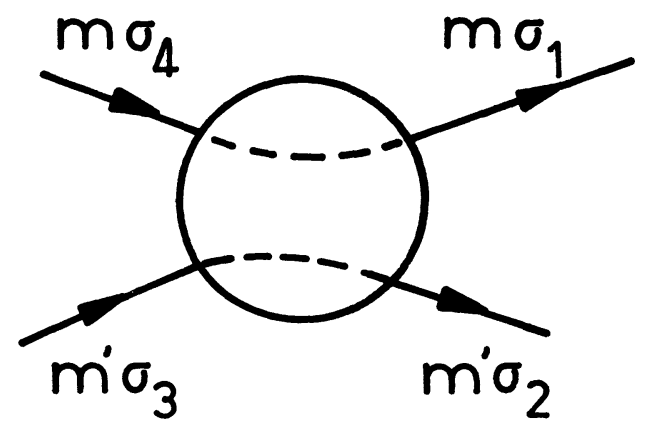

Fig. 8. - A schematic representation of an electron-electron interaction diagram : the dotted line indicates the continuous electron path along which $m$ is conserved.

with coefficients independent of $m$ and $m^{\prime}$. The most general combination of that type invariant under spin and orbital rotation is

$$
\begin{array}{r}
\frac{1}{2} A C_{m \sigma}^{*} C_{m^{\prime} \sigma^{\prime}}^{*} C_{m^{\prime} \sigma^{\prime}} C_{m}+\frac{1}{2} B C_{m \sigma_{1}}^{*} C_{m^{\prime} \sigma_{1}^{\prime}}^{*} C_{m^{\prime} \sigma_{2}^{\prime}} \times \\
\times C_{m \sigma_{2}} \mathbf{s}_{\sigma_{1} \sigma_{2}} \cdot \mathbf{s}_{\sigma_{1}^{\prime} \sigma_{2}^{\prime}} .
\end{array}
$$

It involves two independent parameters $A$ and $B$, instead of the $(2 l+1)$ parameters expected in the most general case : symmetry arguments (here $m$ conservation) are quite efficient in reducing uncertainties as $T \rightarrow 0$ ! (of course, such a symmetry only holds if the Hamiltonian (18) is valid before the Kondo cross over starts, i.e. if $T_{\mathrm{K}} \gg E_{ \pm}$: otherwise, all five Landau interaction coefficients would reappear).

When the cut off $D \rightarrow 0$, the interaction terms (32) are small (they describe the approach to the fixed point). In the spirit of the Landau theory, we treat them within first order perturbation theory, equivalent to a Hartree Fock approximation (remember that the coefficients are fully renormalized). The corresponding linearization is achieved by contracting one $C$ and one $C^{*}$ in all possible ways. Let us assume for simplicity that the spin distribution is polarized in the $z$ direction. Then (32) is replaced by

$$
\begin{aligned}
H_{\mathrm{eff}}=\sum_{m \sigma \neq m^{\prime} \sigma^{\prime}}\left(A+B \sigma \sigma^{\prime}\right) \bar{n}_{m \sigma} n_{m^{\prime} \sigma^{\prime}}- & \\
& -\frac{B}{2} \sum_{m \sigma} \bar{n}_{m \sigma} n_{m,-\sigma}
\end{aligned}
$$

$n_{m \sigma}=C_{m \sigma}^{*} C_{m \sigma}$ is the number operator, $\bar{n}_{m \sigma}$ its expectation value. $\sigma= \pm 1 / 2$ is the component of $s_{z}$. The last term of (33) arises from the contraction of the first and third operators in (32) (or the second and fourth). The physical origin of that term is clear if we consider the average exchange energy between two electrons $m$ and $m^{\prime}$ with opposite spin

- when $m \neq m^{\prime}$, the energy $(-B / 4)$ is the average between singlet and triplet configurations,

- when $m=m^{\prime}$, the energy $(-3 B / 4)$ is that of the singlet (the triplet is forbidden by the exclusion principle).

From (33), we deduce the scattering phase shift $\delta_{m \sigma}$ of an incoming electron with quantum numbers $m \sigma$. That phase shift depends on the energy $z$ and on the electron distribution $\delta \bar{n}_{m^{\prime} \sigma^{\prime}}$ measured from the ground state. Since $H_{\text {eff }}$ is small, $\delta$ is evaluated within Born approximation : it is linear in $H_{\text {eff }}$. From (33) it will have the form :

$$
\begin{aligned}
& \delta_{m \sigma}(z)=\delta_{0}+\alpha z+ \\
& +\sum_{m^{\prime} \neq m}\left[(\varphi+\psi) \delta \bar{n}_{m^{\prime} \sigma}+(\varphi-\psi) \delta \bar{n}_{m^{\prime},-\sigma}\right] \\
& \quad+\sum_{m}(\varphi-3 \psi) \delta \bar{n}_{m,-\sigma}
\end{aligned}
$$

(34) replaces (22). The two interaction parameters $\varphi$ and $\psi$ are related to $A$ and $B$. The Fermi level phase shift $\delta_{0}$ follows either from the Friedel sum rule or from suitably modified electron hole symmetry considerations (see [7]). Finally, $\alpha \sim 1 / T_{\mathrm{K}}$ provides the energy scale.

In order to dispose of the remaining two parameters $\varphi$ and $\psi$, we make use of our universality requirements. Weak universality requires that $\delta$ be invariant if the energy $z$ and all chemical potentials move by the same amount. Let $v=\rho /(2 l+1)$ be the density of states per channel. That condition yields

$$
\alpha+v[4 l \varphi+\varphi-3 \psi]=0 .
$$

Since scattering never changes $m$, strong universality also holds : $\delta_{m \sigma}$ must be unchanged if the Fermi level $\mu_{m^{\prime}}$ of another channel is moved. Thus $2 \varphi=0$. Altogether, we are left with

$$
\varphi=0, \quad \psi=\alpha / 3 v
$$

All interaction parameters are determined : the low temperature behaviour is universal.

From the phase shift, we easily find the physical properties using the approach of ref. [6]. For the specific heat $C_{v}$, the change of population $\delta n_{m \sigma}$ due to a temperature increase is zero : the increment due to the impurity is given by

$$
\frac{\Delta C_{\mathrm{v}}}{C_{\mathrm{v}}}=\frac{\alpha}{\pi v} .
$$

For the net spin susceptibility $\chi$, the Fermi levels for 
up and down spins are shifted by opposite amounts, $\pm \delta \mu$. The phase shift at Fermi level becomes

$$
\delta_{m \sigma}^{\mathrm{F}}= \pm[\alpha \delta \mu+v(4 \psi+3 \psi) \delta \mu] .
$$

Hence an increment in $\chi$

$$
\frac{\Delta \chi}{\chi}=\frac{1}{\pi v}[\alpha+(4 l+3) v \psi] .
$$

On combining (35), (36) and (37), we finally obtain

$$
\frac{\Delta \chi / \chi}{\Delta C_{\mathrm{v}} / C_{\mathrm{v}}}=\frac{2}{3}(2 l+3) .
$$

We thus recover by very simple arguments the result found by Yoshimori [3] and Mihaly-Zawadowski [5] (who analyzed the structure of a full perturbation theory).

To conclude our analysis, let us consider briefly the effects of a cubic anisotropy. The $m$ index is replaced by the five components $\alpha$ of the $E$ and $T_{2}$ representation. Invariance under the cubic group implies that $\alpha$ is conserved at each vertex (Wigner Eckardt theorem) but $\Gamma_{\mathrm{E}}$ and $\Gamma_{\mathrm{T}}$ are different. Consequently, the Landau expansions at $T \ll T_{\mathrm{K}}$ for the energy and for the phase shifts still have the form (33) and (34) - but the number of phenomenological parameters is much larger, namely

$$
\begin{array}{ll}
\delta_{0 \mathrm{E}}, \delta_{0 \mathrm{~T}} ; & \varphi_{\mathrm{TT}}, \varphi_{\mathrm{TE}}, \varphi_{\mathrm{EE}} \\
\alpha_{\mathrm{E}}, \alpha_{\mathrm{T}} ; & \psi_{\mathrm{TT}}, \psi_{\mathrm{TE}}, \psi_{\mathrm{EE}} .
\end{array}
$$

Once again universality reduces that number somewhat. The phase shift must be invariant under a relative translation of chemical potentials in different channels (TT, EE, ET pairs) - hence the conditions

$$
\varphi_{\mathrm{TT}}=\varphi_{\mathrm{EE}}=\varphi_{\mathrm{ET}}=0 .
$$

Moreover $\delta_{\mathrm{E}}$ and $\delta_{\mathrm{T}}$ must be invariant under a global translation of $z$ and all the $\mu_{\alpha}$ : hence two extra conditions

$$
\alpha_{\mathrm{E}}=3 v \psi_{\mathrm{EE}}, \quad \alpha_{\mathrm{T}}=3 v \psi_{\mathrm{TT}} .
$$

In the end, there remains two unknown parameters : the ratio $\alpha_{\mathrm{E}} / \alpha_{\mathrm{T}}$ and the interaction coefficient $\psi_{\mathrm{TE}}$. The low temperature behaviour will thus be non universal. For instance, the Wilson ratio will depart from its value (38).

In the most general case, we can say nothing more. If however the anisotropy has become very large by the time the Kondo cross over starts, a new universality is established. Then, one channel takes over, for instance $\mathrm{E}$, and we can neglect the other one. Only $\alpha_{\mathrm{E}}, \varphi_{\mathrm{EE}}, \psi_{\mathrm{EE}}$ survive, and there is no unknown left. The calculation is the same as before, except that $(2 l+1)$ is replaced by the number $n$ of relevant orbital channels ( 2 for an $\mathrm{E}$ level, 3 for a $\mathrm{T}_{2}$ level). The
Wilson ratio is then

$$
\frac{\Delta \chi / \chi}{\Delta C_{\mathrm{v}} / C_{\mathrm{v}}}=\frac{2}{3}(n+2) .
$$

Such a strong anisotropy may be a consequence of - either the initial conditions $\left(\Gamma_{\mathrm{E}}\right.$ and $\Gamma_{\mathrm{T}}$ very different, as in the cases $n_{\mathrm{d}_{0}}=3$ or 8 ),

- or the growth of an initially weak anisotropy during scaling. In order to assess the amount of universality, a detailed discussion is necessary in each given case.

6. Impurities with a single d-electron (or hole). The problem was studied long ago by Coqblin and Schrieffer [18]. It might correspond for instance to a $\mathrm{Ce}$ (or $\mathrm{Yb}$ ) impurity. In first approximation, let us ignore crystal field or spin orbit effects. Then $\varepsilon_{0}$ contains only one multiplet, corresponding to $L=l$, $S=1 / 2$.

In such a case, the Schrieffer Wolff transformation proceeds directly to the low temperature $S$-d model (no splitting of $H_{\mathrm{at}}^{0}$ to worry about). If we neglect splittings in the virtual ionization energies $E_{ \pm}$, the VS symmetry (7) holds. That symmetry is exact if we consider only ionization to $n_{\mathrm{d}}=0$ (there is only one such state) ; it is approximate if we include ionization to $n_{\mathrm{d}}=2$. We assume here that such a symmetry is valid, and we write the initial scattering Hamiltonian as $\left({ }^{8}\right)$

$$
a_{0} \sum_{m \sigma} C_{m \sigma}^{*} C_{m \sigma}+b_{0} \sum_{\substack{m \sigma \\ m^{\prime} \sigma^{\prime}}} C_{m \sigma}^{*} C_{m^{\prime} \sigma^{\prime}} a_{m^{\prime} \sigma^{\prime}}^{*} a_{m \sigma}
$$

(see (7)). We have shown that the VS symmetry was stable upon scaling; the renormalized coefficients $a$ and $b$ obey (14). We are then in a position to carry our program : (i) nature and stability of the strong coupling limit (ii) phenomenological description of the $T \rightarrow 0$ limit.

6.1 THE ISOTROPIC STRONG COUPLING LIMIT. When $b \rightarrow+\infty, \mathrm{p}$ conduction electrons are trapped on the impurity site. The corresponding ground state is such as to maximize the resonance between those $p$ electrons and the single d-electron. Let us introduce again the notation $\alpha=m, \sigma$. Any doubly occupied channel is lost for such a resonance. Thus it is energetically advantageous to distribute the $(p+1)$ electrons among $(p+1)$ different channels; the Hamiltonian (40) will then interchange the position of the d-electron among these $(p+1)$ channels. For a given choice of the occupied channels, the eigenstates are easily found :

- $\mathrm{p}$ states have energy $(\mathrm{p} a+b)$,

- one state has energy $(\mathrm{p} a-\mathrm{p} b)$.

$\left({ }^{8}\right)$ That operator acts only on the $n_{\mathrm{d}}=1$ manifold. Nevertheless, it is convenient to use the fermion operator $a_{m \sigma}$ in order to describe scattering of the impurity from state $(m \sigma)$ to $\left(m^{\prime} \sigma^{\prime}\right)$. 
From (14) and (15), we know that $b>0$ : the singlet state is lowest (it corresponds to a d-electron symmetrically distributed between channels).

For arbitrary $p$, we still have the degeneracy due to the choice of occupied channels. The lowest energy will correspond to $\mathrm{p}$ as large as possible : all channels are occupied by one electron, i.e.

$$
\mathrm{p}+1=M=2(2 l+1) \text {. }
$$

There is only one way to do so : the strong coupling ground state is non degenerate, with an energy

$$
(M-1)(a-b)=-b \frac{M^{2}-1}{M}
$$

The corresponding fixed point is stable, with the usual Fermi liquid behaviour $\left({ }^{9}\right)$.

6.2 THE FERMI LIQUID DESCRIPTION. - The same index $m \sigma$ characterizes the conduction electron (full line in the diagrams) and the impurity state (dotted line). In view of (40), the basic vertex has a new symmetry depicted on figure $9 a: m \sigma$ is conserved

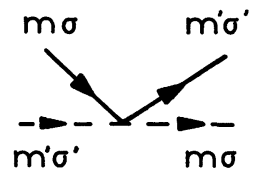

(a)

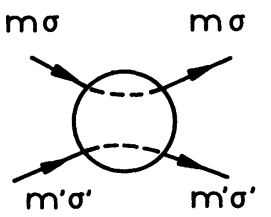

(b)
Fig. 9. - (a) The basic interaction vertex for VS symmetry : $m \sigma$ is exchanged between the full and dotted lines. $(b)$ The effective electron-electron interaction vertex : the $m \sigma$ quantum numbers are conserved along an alternation of full and dotted lines.

from full to dotted line. It follows that any electronelectron interaction vertex should have the form of figure $9 b(m, \sigma$ are conserved along an alternation of full and dotted lines). The effective interaction (23) reduces to

$$
\frac{1}{2} B \sum_{m \sigma \neq m^{\prime} \sigma^{\prime}} C_{m \sigma}^{*} C_{m^{\prime} \sigma^{\prime}}^{*} C_{m^{\prime} \sigma^{\prime}} C_{m \sigma}
$$

It depends on a single parameter $B$. The symmetry arguments are here very powerful! Following the same discussion as for (32), we write the phase shift in the form

$$
\delta_{m \sigma}(z)=\delta_{0}+\alpha z+\varphi\left[\sum_{m^{\prime}} \delta n_{m^{\prime},-\sigma}+\sum_{m^{\prime} \neq m} \delta n_{m^{\prime} \sigma}\right]
$$

The phenomenological parameter $\varphi$ is related to $B$.

(9) The same conclusion holds whenever VS symmetry holds for arbitrary $n_{\mathrm{d}_{0}}$ : the ground state is obtained by distributing evenly $n_{\mathrm{d}_{0}}$ d-electrons and $\left(M-n_{\mathrm{d}_{0}}\right)$ conduction electrons among all channels, one per channel.
In the present case, strong universality does not hold, since the interaction (40) allows a conduction electron to jump from one channel to any other. Only weak universality is valid, but it is enough for a single parameter. Requiring that $\delta$ be invariant under simultaneous translation of $z$ and $\mu$, we find

$$
\alpha+v \varphi(4 l+1)=0 \text {. }
$$

The calculation of $C_{\mathrm{v}}$ and $\chi$ is then straightforward. We find the following expression for the Wilson ratio

$$
\frac{\Delta \chi / \chi}{\Delta C_{\mathrm{v}} / C_{\mathrm{v}}}=1-\frac{v \varphi}{\alpha}=\frac{2(2 l+1)}{4 l+1} .
$$

More generally, if there are $n$ equivalent orbital channels, (43) should be replaced by

$$
\frac{\Delta \chi / \chi}{\Delta C_{\mathrm{v}} / C_{\mathrm{v}}}=\frac{2 n}{2 n-1}
$$

The low temperature behaviour is again universal.

6.3 THE INFLUENCE OF ANISOTROPY. - In the presence of a cubic field $(l=2)$, the impurity ground state splits into a triplet $T_{2}$ and a doublet $E$. The triplet is lowest for $n_{\mathrm{d}_{0}}=1$, the doublet for $n_{\mathrm{d}_{0}}=9$. We have shown in section 2.4 that spin exchange scattering was important only in the same state as the impurity, i.e. $\Gamma_{\mathrm{TT}}$ for a $\mathrm{T}_{2}$ ground state and $\Gamma_{\mathrm{EE}}$ for an $\mathrm{E}$ ground state. The other couplings do exist but they are small. We neglect them altogether for simplicity. We shall choose the $\mathrm{E}$ ground state as an example, but our discussion would easily be transposed to the $\mathrm{T}_{2}$ state.

Remember that the combination of an E impurity with an $\mathrm{E}$ electron gives three orbital states

$$
E \otimes E=A_{1}+A_{2}+E .
$$

Hence six independent parameters in the Schrieffer Wolff hamiltonian. The corresponding weak coupling scaling equations are written in ref. [13] : they are fairly complicated. Physically, the complication arises from a mixture of spin and orbital Kondo effects. Both the spin and orbital structure of the impurity provide a memory for conduction electron scattering, and the corresponding singularities are deeply interwoven.

The nature of the ground state in the strong coupling limit depends on the ratios of the above six constants. In the absence of a quantitative treatment of the cross over region, we can only guess that the couplings will evolve in such a way that the ground state is non degenerate. Such a statement is far from obvious, albeit likely. We take it as granted, and we proceed to study the Fermi liquid description at $T \ll T_{\mathrm{K}}$.

Since in the Schrieffer Wolff hamiltonian we retained only the $\mathrm{E}$ electron states, the low temperature Landau hamiltonian (23) will only involve $C_{\alpha \sigma}$ opera- 
tors belonging to that representation. The number of independent interaction parameters in (23) is the number of states one can build with two $E$ electrons (spin 1/2). Since the electrons are indistinguishable (they belong to the same conduction band), there are three such states

$$
{ }^{0} \mathrm{~A}_{1},{ }^{1} \mathrm{~A}_{2},{ }^{0} \mathrm{E} \text {. }
$$

We conclude that in the expansion of the phase shift for $\mathrm{E}$ electrons there are three interaction parameters (in addition to $\delta_{0}$ and $\alpha$ ). In general, strong universality does not hold, and weak universality provides only one relationship between these parameters. Thus, there remain two unknown in the low temperature properties : they are not universal (e.g. the Wilson ratio has no prescribed value).

In limiting cases, one may invoke additional symmetries, for instance VS symmetry which leads back to (44) (the number of orbital channels is here $n=2$ ). Similarly, a pure spin Schrieffer Wolff Hamiltonian would be

$$
\text { S. } \mathbf{s}_{\sigma \sigma^{\prime}} C_{\alpha \sigma}^{*} C_{\alpha \sigma^{\prime}}
$$

(where $\alpha$ is the $\mathrm{E}$ doublet component). The situation is then equivalent to an orbital singlet, and (39) applies. Unfortunately, such a symmetry is completely unrealistic as the spin and orbital couplings are comparable. Other symmetries may be helpful. For instance, if the $A_{1}$ and $E$ orbital states are degenerate in the Schrieffer Wolff hamiltonian, the E doublet can be treated as a fictitious spin $1 / 2$, and one parameter disappears at low temperature.

These few examples show that the low temperature universality reflects the symmetries above the cross over. If all the original couplings are comparable, we cannot avoid the full complexity of the Landau phenomenological description at low temperature. In a fancy language, the direction of approach to the fixed point depends on where we started (there are several equally relevant variables near that fixed point). As a first approximation, VS symmetry is the best choice.

6.4 THE INFLUENCE OF SPIN ORBIT COUPLING. In the presence of spin orbit coupling (but without crystal field), the one electron impurity state splits in two components, with total momentum

$$
J=l \pm 1 / 2
$$

separated by an energy $\lambda$. The hybridization term conserves $m$ and $\sigma$, and thus $J$ and $J_{z}=\tau$. Let us take the $J$ eigenstates as a basis. Assuming again VS symmetry, we may write the effective Schrieffer Wolff Hamiltonian as

$$
\begin{aligned}
H_{\text {eff }}=\sum_{\tau} \lambda d_{J_{1} \tau}^{*} d_{J_{1} \tau}+\sum_{J_{\tau}} a C_{J_{\tau}}^{*} & C_{J_{\tau}}+ \\
& +\sum_{\substack{J_{\boldsymbol{J}} \tau \\
J^{\prime} \tau^{\prime}}} b C_{J_{\tau}}^{*} C_{\boldsymbol{J}^{\prime} \tau^{\prime}} d_{\boldsymbol{J}^{\prime} \tau^{\prime}}^{*} d_{\boldsymbol{J} \tau}
\end{aligned}
$$

( $J_{0}$ corresponds to the ground state, $J_{1}$ to the excited state). The central issue is now to compare $T_{\mathbf{K}}$ with $\lambda$.

(i) If $T_{\mathbf{K}} \gg \lambda$, we may neglect the splitting $\lambda$. The $J_{0}$ and $J_{1}$ states are on the same footing, and we have $n_{1}=2(2 l+1)$ equivalent channels. The result (44) applies with $n=n_{1}$.

(ii) If $T_{\mathrm{K}} \ll \lambda$, only the ground state $J_{0}$ participates in the Kondo cross over. We again have a VS symmetric problem, but with a smaller number of channels, $n_{2}=2 J_{0}+1$. (44) again applies, with $n=n_{2}$.

(iii) If $T_{\mathrm{K}} \sim \lambda$, we loose VS symmetry. The Wilson ratio will be a function of $\left(T_{\mathrm{K}} / \lambda\right)$, intermediate between regimes (i) and (ii) : universality is lost.

The interplay of the Kondo cross over and the splittings of $H_{\mathrm{at}}^{0}$ is thus clearly demonstrated.

7. Conclusion. - Our paper does not pretend to be exhaustive. We only tried to give general arguments that describe the hierarchy of cross overs, the relevant parameters, the relationship between symmetries above and below $T_{\mathrm{K}}$, etc... Our purpose was to lay the ground for a detailed study of individual cases. On a few examples, we have shown that very simple considerations give access to universality properties at low temperature : that should provide the language in which to describe actual physical systems. A detailed analysis is required in each specific case.

Acknowledgements. - This work is already old. In the course of time, we benefited from discussions with numerous physicists. We would like to thank especially A. Zawadowski for his comments, and P. Lloyd and D. Cragg for their beautiful numerical work which helped substantiating some of our speculations.

\section{References}

[1] Following the original article of Anderson, P. W., Phys. Rev. 124 (1961) 41, the lack of rotational invariance was successively cured in the papers of

Caroli, B., Caroli, C., Fredkin, D., Phys. Rev. 178 (1969) 599 ;

Caroli, B., Lederer, P., Saint James, D., Phys. Rev. Lett. 23 (1969) 700 ;
Dworin, L., Narath, A., Phys. Rev. Lett. 25 (1970) 1287.

[2] See the exhaustive review article of HIRST, L. L., Adv. in Phys. 27 (1978) 231, and references therein.

[3] Yoshimori, A., Progr. Theor. Phys. 55 (1976) 67.

[4] Yamada, K., Progr. Theor. Phys. 53 (1975) 1970 and 54 (1975) 316.

Yosida, K., Yamada, K., Progr. Theor. Phys. 53 (1975) 1286. 
[5] Mihaly, L., Zawadowski, A., J. Physique Lett. 39 (1978) L-483.

[6] Nozières, P., J. Low Temp. Phys. 17 (1974) 31.

Proceedings of LT14, Vol. 5 (North Holland) 1975.

[7] NozIÈres, P., J. Physique 39 (1978) 1117.

[8] See for instance Condon, E. E., Shortley, G. H., The Theory of Atomic Spectra (Cambridge Univ. Press) 1970, Chap. 7.

[9] See for instance Blandin, A., J. Appl. Phys. 39 (1968) 1285.

[10] Anderson, P. W., J. Phys. C 3 (1970) 2346 ;

Yuval, G., Anderson, P. W., Phys. Rev. B 1 (1970) 1522.

Anderson, P. W., Yuval, G., Hamann, D. R., Phys. Rev. B 1 (1970) 4664;

A critical review may be found in ANDERson, P. W., Comments Solid State Phys. 5 (1973) 73.

[11] Wilson, K. G., Collective properties of Physical Systems, Nobel Symposium 24 (Academic Press) 1974 ; Rev. Mod. Phys. 47 (1975) 773.

The Wilson's approach has been generalized to the asymmetric Anderson model $(l=0)$ by
Krishnamurthy, H. R., Wilson, K. G., Wilkins, J. W., Phys. Rev. Lett. 35 (1975) 1101, and

in Valence Instabilities and Narrow Band Related Phenomena, edited by Parks, R. D. (Plenum, New York) 1977, p. 177.

The qualitative nature of scaling in that case has been extensively discussed by Haldane, F. D. M., Phys. Rev. Lett. 40 (1978) 416, J. Phys. C 11 (1978) 5015.

[12] Cornut, B., CoQblin, B., Phys. Rev. B 5 (1972) 4541.

[13] Cragg, D. M., Lloyd, P., Nozières, P., submitted to $J$. Phys. C.

[14] Cragg, D. M., Lloyd, P., J. Phys. C 11 (1978) L-597 and J. Phys. C 12 (1979) L-215.

[15] Cragg, D. M., Lloyd, P., J. Phys. C 12 (1979) 3301.

[16] Abrikosov, A. A., Migdal, A. A., J. Low Temp. Phys. 3 (1970) 519.

[17] Fowler, M., Zawadowski, A., Solid State Commun. 9 (1971) 471.

[18] CoQblin, B., Schrieffer, J. R., Phys. Rev. 185 (1969) 847. 